\title{
Adaptive Fixed-Time Trajectory Tracking Control for Underactuated Hovercraft with Prescribed Performance in the Presence of Model Uncertainties
}

\author{
Mingyu Fu, Tan Zhang ${ }^{D}$, Fuguang Ding, and Duansong Wang \\ College of Intelligent Systems Science and Engineering, Harbin Engineering University, Harbin 150001, China \\ Correspondence should be addressed to Tan Zhang; zhangtantan@hrbeu.edu.cn
}

Received 2 November 2020; Revised 14 March 2021; Accepted 26 March 2021; Published 12 April 2021

Academic Editor: Chongyang Liu

Copyright (c) 2021 Mingyu Fu et al. This is an open access article distributed under the Creative Commons Attribution License, which permits unrestricted use, distribution, and reproduction in any medium, provided the original work is properly cited.

\begin{abstract}
This paper develops an adaptive fixed-time trajectory tracking controller of an underactuated hovercraft with a prescribed performance in the presence of model uncertainties and unknown time-varying environment disturbances. It is the first time that the proposed method is applied to the motion control of the hovercraft. To begin with, based on the hovercraft's four degrees of freedom (DOF) model, the virtual control laws are designed using an error transforming function and the fixed-time stability theory to guarantee that the position tracking errors are constrained within the prescribed convergence rates and minimum overshoot. In addition, by combining the Lyapunov direct method and the adaptive radial basis function neural network (ARBFNN), the actual control laws are designed to ensure that the velocity tracking errors converge to a small region containing zero while handling model uncertainties and external disturbances effectively. Finally, all tracking errors of the closed-loop system are uniformly ultimately bounded and fixed-time convergent. Results from a comparative simulation study verify the effectiveness and advantage of the proposed method.
\end{abstract}

\section{Introduction}

The underactuated hovercraft as shown in Figure 1 possesses a flexible skirt around the bottom of it to seal the cushion air [1]. It can be lifted to the sail by a large enough air cushion force to obtain a unique amphibious performance. Therefore, the hovercraft has a higher speed than normal surface vessels. In recent years, because of its special amphibious performance, the hovercraft has been getting increasing attention in oceanic research work, for instance, oceanic resources exploration, scientific investigation, ocean expeditions, military missions, transportation, rescue, and other fields [2].

What is particularly noteworthy is that the hovercraft in navigation possesses very little contact with the sailing surface; therefore, it has smaller righting moments than normal surface vessels. It is easy to generate a large roll angle and large drift angle when turning the rudder so that the hovercraft runs in a dangerous situation. The air cushion force's lateral component under a large roll angle results in the hovercraft drifting sideways and losing course stability [3-5]. The roll motion occurs always when the hovercraft is turning and is more strenuous with the increase in the turn rate and drift angle. Regarding that the above dangerous sailing mechanism is closely relevant to the roll motion of the hovercraft; a four-DOF motion mathematical model of a hovercraft by taking the roll degree of freedom into account is established in this paper, which is closer to the sailing characteristics of the actual hovercraft than the three-DOF model.

Usually, the hovercraft has the characteristics of the typical underactuated vessel. The major difficulty for motion control of the underactuated surface vessels is that the lateral motion is not controlled directly, namely, the number of DOF is more than the number of independent actuators in the underactuated system. The challenge to control underactuated vessels is how to apply two independent actuators to regulate three or more DOFs' motions under 




FIGURE 1: Reference frame of the hovercraft.

unknown dynamic environments. Over the past two decades, for overcoming the above control problems, various control strategies have been presented by the endeavours of many research workers, and significant achievements have been achieved. In [6], an adaptive fuzzy stabilization control method was proposed to carry out trajectory tracking control of the underactuated surface vessel in the presence of unknown time-varying environment disturbances. Considering the robustness of the underactuated vessel's control, the literature [7] adopted the biologically inspired method to perform motion control of an underactuated surface vessel and used the single-layer neural network to handle the unknown dynamics. An adaptive dynamic sliding mode control method for trajectory tracking control of an underactuated underwater unmanned vehicle was proposed in [8], but the proposed adaptive sliding mode control method requires some assumptions, such as the existence of the first derivative of external disturbance and control input. In [9], the combination of neural network and the terminal sliding mode was used to design a finite-time trajectory tracking controller for an underactuated hovercraft. A $H_{\infty}$ observer-based fuzzy controller design for a vessel dynamic positioning system was investigated in [10], and the simulation results show that this method is effective for both known/unknown premise variable cases.

Unfortunately, one important problem, the transient and steady error performance constraint playing a significant role in practice, is not addressed in the above papers. The transient performance constraints can guarantee that high-speed hovercraft avoids collision hazards and provides stability simultaneously. Recently, a performance constraint technique was proposed in [11-13]. This technique can guarantee that the transient errors converge at a designed exponential rate with a predesigned maximum overshoot. Now, the prescribed performance control strategy has been applied to many practical system controls, such as servo system [14], air vehicles $[15,16]$, and robots $[17,18]$. With the further research of scientific researchers, a series of improved prescribed performance control technology has been proposed in [19-23] to constrain the transient and steady-state performance of the control system. An envelope-constraint-based tracking control method for airbreathing hypersonic vehicles with unknown nonaffine formulations is proposed in [19], and the desired transient performance is guaranteed for velocity and attitude tracking errors using envelope constraint. In [21], a novel performance function without the information of the initial error is proposed for limiting the tracking error of hypersonic flight vehicles into a prescribed range. A new prescribed performance control approach utilizing transformed errors instead of initial tracking errors is proposed for uncertain nonlinear dynamic systems in [22]. For the velocity dynamics of air-breathing hypersonic vehicles, an adaptive neural controller containing only one neural network is addressed using the prescribed performance control method in [23]. The highlights of the method in [23] are that the presented control method has a concise control architecture and low computational cost. Subsequently, the prescribed performance technique has been applied practically to the motion control of the underactuated vessels. For example, a robust adaptive controller was presented to perform the motion control of the underactuated ship with prescribed performance in the presence of model uncertainties and external disturbances [24]. In [25], the transient and steadystate performance was also featured for trajectory tracking control of underactuated underwater vessels.

On the other hand, the controller designed in the practical engineering applications of the ship only guarantees that the stability of the system is not to make much sense. The system convergence speed is always an important index to evaluate the designed controller's performance. In the last few years, the control technique for specifying the system convergence time was proposed; for example, one type of delayed memristor-based fractional-order neural networks based on the finite-time stability problem was studied in [26]. A novel adaptive fixed-time controller was 
designed to perform output tracking problems of a class of multiinput multioutput nonlinear systems in the presence of system uncertainties [27]. A fixed-time sliding mode manifold was proposed [28], and the convergence time of the sliding mode manifold is shown to be independent of the initial conditions of the control system. In [29], a nonsingular fixed-time fast terminal sliding mode manifold with fixed-time convergence was presented, and the preestablished convergence time is also developed; unfortunately, the control law designed is discontinuous.

Inspired by the abovementioned observations, a nonlinear trajectory tracking control method for an underactuated hovercraft is presented that ensures a prescribed performance, specifies the system convergence time, and handles the system uncertainties. Incorporating the prescribed performance and specified system convergence time simultaneously in the trajectory tracking control design of the underactuated hovercraft has been unprecedented. Accordingly, the main contributions of this paper are summarized as follows:

(1) Unlike the existing trajectory tracking control methods of the underactuated hovercraft in [9], the presented control strategy avoids the dangerous navigation situation when the hovercraft sails in a narrow channel. Compared with the barrier Lyapunov function method [30], one of the advantages of the prescribed performance method is that it can increase the space of the initial feasible solution of the system and ensure that the tracking error of the system can converge to any small region set in advance.

(2) To solve the unknown dynamic model uncertainties of the controlled system and external disturbances, an ARBFNN is applied to the trajectory tracking control scheme that is simple and easy to implement in practice.

(3) By selecting the control parameters appropriately, the convergence time that is independent of the initial values of the controlled system can be prespecified to guarantee a fast convergence performance of the nonlinear trajectory tracking system.

(4) It is proven that, by applying the presented controller, all tracking errors of the closed-loop system are uniformly ultimately bounded and uniformly fixed-time convergent despite the presence of system uncertainties.

This paper is arranged as follows. The preliminaries and problem formulation are given in Section 2. Section 3 is devoted to the design of the adaptive neural-based fixedtime trajectory tracking controller for the hovercraft with prescribed performance. Numerical simulation results are shown in Section 4. Section 5 concludes the work of this paper.

\section{Preliminaries and Problem Formulation}

\subsection{Preliminaries}

Notation. Throughout this paper, $(\cdot)^{T}$ denotes the transpose of a matrix $(\cdot),\|\cdot\|$ represents the Euclidean norm of a vector, $|\cdot|$ signifies the absolute value of a scalar, $\lambda_{\min }(\cdot)$ and $\lambda_{\max }(\cdot)$ mean the minimum and maximum eigenvalues of a given matrix $B \in R^{n \times n}$, respectively, $U_{1} \cup U_{2}$ denotes the union of interval $U_{1}$ and interval $U_{2}$, and sign (.) denotes the signum function.

Lemma 1 (see [31]). For any real numbers $x_{i} \in R$, $i=1,2, \ldots, n$, we can obtain the following inequalities:

$$
\sum_{i=1}^{n}\left|x_{i}\right|^{p} \geq\left\{\begin{array}{l}
\left(\sum_{i=1}^{n}\left|x_{i}\right|\right)^{p}, \quad 0<p \leq 1, \\
n^{1-p}\left(\sum_{i=1}^{n}\left|x_{i}\right|\right)^{p}, \quad 1<p<+\infty .
\end{array}\right.
$$

Lemma 2 (see [32]). For any continuous unknown real function $f(x): R^{m} \longrightarrow R$, the radius basis function neural network (NN) can be employed to approximate it over the compact $\Omega \subseteq R^{m}$ as follows:

$$
f(x)=w^{* T} h(x)+\delta(x)
$$

where $x \in R^{m}$ is the input vector of $N N$, $w^{*}=\left[w_{1}^{*}, \ldots, w_{n}^{*}\right]^{T} \in R^{n}$ denotes the $N N$ ideal bounded weight vector, $n$ denotes the hidden note number of $N N$, and $\delta(x)$ represents the ideal approximation error with satisfying $|\delta(x)| \leq \bar{\delta}$, where $\bar{\delta}$ is a constant. The ideal weight value can be determined by the following equation:

$$
w^{*}=\arg \min _{\widehat{w}}\left\{\sup _{x \in \Omega}\left|f(x)-\widehat{w}^{T} h(x)\right|\right\},
$$

where $\widehat{w}$ is the estimation of the ideal weight value $w^{*}$, which is usually calculated by the adaptive updating law based on the Lyapunov stability theorem. The radius basis function vector is $h(x)=\left[h_{1}(x), \ldots, h_{n}(x)\right]^{T}: \Omega \longrightarrow R^{n}$, and $h_{i}(x)$ are selected as the Gaussian function in the following form:

$$
h_{i}(x)=\exp \left(-\frac{\left\|x-\phi_{i}\right\|^{2}}{\varepsilon_{i}^{2}}\right), \quad(i=1,2, \ldots, n),
$$

where $\phi_{i} \in R^{m}$ and $\varepsilon_{i} \in R$ denote the centre and width of the radius basis function, respectively.

Definition 1. Considering the following first-order system (5), if the system is finite-time stable and the upper bound of convergence time is independent of the initial values of the control system, the system is fixed-time stable:

$$
\dot{x}=f(x)+\tau+d,
$$

where $x \in R$ and $\tau \in R$ are the state and input of system (5), respectively, $f(x)$ is a known continuous function, and $d$ is an unknown continuous bounded function which signifies model uncertainties or/and external disturbances.

Lemma 3 (see [33]). Considering system (5), if there exists a Lyapunov function $V(x)$ which satisfies 


$$
\dot{V}(x) \leq-\alpha V^{p}(x)-\beta V^{q}(x)
$$

where $\alpha, p, \beta$, and $q$ are positive constants and satisfy $0<p<1<q$, system (5) is fixed-time stable and the convergence time can be estimated as follows:

$$
T \leq \frac{1}{\alpha(1-p)}+\frac{1}{\beta(q-1)} .
$$

Lemma 4 (see [34]). In the light of Lemma 3, if there is a positive bounded function $\Delta$ and a Lyapunov function $V(x)$ which satisfy

$$
\dot{V}(x) \leq-\alpha V^{p}(x)-\beta V^{q}(x)+\Delta,
$$

where $\alpha, p, \beta$, and $q$ are positive constants and satisfy $0<p<1<q$, the system state can converge to $D=\left\{x: V(x) \leq \min \left\{\left(\Delta / \alpha \theta_{p}\right)^{1 / p},\left(\Delta / \beta \theta_{q}\right)^{1 / q}\right\}\right\}$ within time $T$ :

$$
T \leq \frac{1}{\alpha\left(1-\theta_{p}\right)(1-p)}+\frac{1}{\beta\left(1-\theta_{q}\right)(q-1)},
$$

where $\theta_{p}, \theta_{q} \in(0,1)$ are positive constants.

2.2. Hovercraft Model. According to [2], the following kinematic model and dynamic model are employed to characterize the four-DOF motions of the hovercraft in Figure 1:

$$
\begin{aligned}
& \left\{\begin{array}{l}
\dot{x}=u \cos \psi-v \sin \psi \cos \phi, \\
\dot{y}=u \sin \psi+v \cos \psi \cos \phi, \\
\dot{\phi}=p \\
\dot{\psi}=r \cos \phi
\end{array}\right. \\
& \left\{\begin{array}{l}
\dot{u}=v r+\frac{F_{x D 0}}{m_{0}}+f_{u}+\frac{\tau_{u}}{m_{0}}, \\
\dot{v}=-u r+\frac{F_{y D 0}}{m_{0}}+f_{v}, \\
\dot{p}=\frac{M_{x D 0}}{J_{x 0}}+f_{p}, \\
\dot{r}=\frac{M_{z D 0}}{J_{z 0}}+f_{r}+\frac{\tau_{r}}{J_{z 0}},
\end{array}\right.
\end{aligned}
$$

with

$$
\begin{aligned}
& f_{u}=\frac{1}{m_{0}}\left(-\Delta m \dot{u}+\Delta m v r+\Delta F_{x D}\right)+\frac{d_{u}(t)}{m_{0}}, \\
& f_{v}=-\frac{1}{m_{0}}\left(\Delta m(\dot{v}+u r)+\Delta F_{y D}\right)+\frac{d_{v}(t)}{m_{0}}, \\
& f_{p}=\frac{1}{J_{x 0}}\left(-\Delta J_{x} \dot{p}+\Delta M_{x D}\right)+\frac{d_{p}(t)}{J_{x 0}}, \\
& f_{r}=\frac{1}{J_{z 0}}\left(-\Delta J_{z} \dot{r}+\Delta M_{z D}\right)+\frac{d_{r}(t)}{J_{z 0}},
\end{aligned}
$$

where $u$ and $v$ denote velocities, $p$ and $r$ denote the angular velocities in the body-fixed frame, $x$ and $y$ represent positions, and $\phi$ and $\psi$ represent attitudes with respect to the earth-fixed frame. The control inputs are represented by $\tau_{u}$ and $\tau_{r}$. The known hull design mass and moment of inertia are signified by $m_{0}, J_{x 0}$, and $J_{z 0} . \Delta m, \Delta J_{x}$, and $\Delta J_{z}$ are viewed as the uncertainty of the ship's mass and moment of inertia. The known part of the current resistance models of the hovercraft, for example, air momentum force, skirt resistance, air resistance, and wave-making resistance, is denoted by $F_{x D 0}, F_{y D 0}, M_{x D 0}$, and $M_{z D 0}$, respectively, that can be determined through wind tunnel test and tank test. $\Delta F_{x D}$, $\Delta F_{y D}, \Delta M_{x D}$, and $\Delta M_{z D}$ are viewed as the uncertainties of the model in hydrodynamic and aerodynamic drag caused by modelling errors. $d_{u}(t), d_{v}(t), d_{p}(t)$, and $d_{r}(t)$ signify external environment resistance. $f_{u}, f_{v}, f_{p}$, and $f_{r}$ contain the total model uncertainties and external environment resistances. $F_{x D 0}, F_{y D 0}, M_{x D 0}$, and $M_{z D 0}$ can be calculated by the following equations:

$$
\begin{aligned}
F_{x D 0}= & F_{x a}+F_{m} \cos \beta+F_{\mathrm{wm}} \cos \beta+F_{\mathrm{sk}} \cos \beta \\
F_{y D 0}= & F_{y a}+F_{m} \sin \beta+F_{\mathrm{wm}} \sin \beta+F_{\mathrm{sk}} \sin \beta+F_{c}, \\
M_{x D 0}= & M_{x a}+F_{m} z_{m} \sin \beta+F_{\mathrm{wm}} z_{\mathrm{wm}} \sin \beta+G h_{m} \tan \phi+F_{\mathrm{sk}} z_{\mathrm{sk}} \sin \beta+F_{c} z_{c}, \\
M_{z D 0}= & M_{z a}+F_{m} x_{m} \sin \beta+F_{m} y_{m} \cos \beta+F_{\mathrm{wm}} x_{\mathrm{wm}} \sin \beta+F_{\mathrm{wm}} y_{\mathrm{wm}} \cos \beta \\
& +F_{\mathrm{sk}} x_{\mathrm{sk}} \sin \beta+F_{\mathrm{sk}} y_{\mathrm{sk}} \cos \beta+F_{c} x_{c},
\end{aligned}
$$


where

$$
\begin{aligned}
F_{x a}= & -0.5 \rho_{a} V_{a}^{2} C_{x a} S_{\mathrm{PP}}, \\
F_{y a}= & -0.5 \rho_{a} V_{a}^{2} C_{y a} S_{\mathrm{LP}}, \\
M_{x a}= & -0.5 \rho_{a} V_{a}^{2} C_{m x a} S_{\mathrm{PP}} l_{c}+F_{y a} z_{a}, \\
M_{z a}= & -0.5 \rho_{a} V_{a}^{2} C_{m z a} S_{\mathrm{HP}} H_{\mathrm{hov}}+F_{y a} x_{a}+F_{x a} y_{a}, \\
F_{m}= & \rho_{a} V_{a} Q \\
F_{\mathrm{wm}}= & C_{\mathrm{wm}} p_{c}^{2} B_{c} / \rho_{w} g, \\
F_{\mathrm{sk}}= & 0.5 V_{a}^{2} C_{\mathrm{sk}}\left(h / l_{\mathrm{sk}}\right)^{-0.34} l_{\mathrm{sk}} S_{c}^{0.5} \\
& +\left(2.8167\left(p_{c} / l_{c}\right)^{-0.259}-1\right) F_{\mathrm{wm}}, \\
F_{c}= & 2 l_{c} \varphi p_{c}\left(0.5 B_{c} \tan \phi+h_{0}\right), \\
M_{G}= & G h_{m} \tan \phi,
\end{aligned}
$$

where $F_{x a}, F_{y a}, M_{x a}$, and $M_{z a}$ denote air resistance, $F_{\mathrm{wm}}$, $F_{m}, F_{c}$, and $F_{\text {sk }}$ denote wave-making resistance, air momentum force, cushion resistance, and skirt resistance, respectively. $C_{x a}, C_{y a}, C_{m x a}, C_{m z a}, C_{\mathrm{wm}}$, and $C_{\mathrm{sk}}$ represent the relevant resistance parameters. $M_{G}$ means roll restoring moment. The length and width of the cushion are represented by $l_{c}$ and $B_{c}$, respectively. $S_{\mathrm{LP}}, S_{\mathrm{PP}}$, and $S_{\mathrm{HP}}$ denote lateral, positive, and horizontal projection areas, respectively. $S_{c}$ and $p_{c}$ are the area and pressure of the cushion, the average clearance for air leakage in static hovering mode is signified by $h, h_{m}$ denotes the metacentric height, $h_{0}$ denotes the initial lifting height, the total length of the skirt is represented by $l_{\text {sk }}$, the flow coefficient is signified by $\varphi, H_{\text {hov }}$ means the hovercraft's height, the air density and water density are signified by $\rho_{a}$ and $\rho_{w}, z_{a}, z_{m}, z_{\mathrm{wm}}, z_{\mathrm{sk}}$, and $z_{c}$ denote heights of each force's acting point with respect to the mass centre of the hovercraft, and $\left(x_{a}, y_{a}\right),\left(x_{m}, y_{m}\right)$, $\left(x_{\mathrm{wm}}, y_{\mathrm{wm}}\right),\left(x_{\mathrm{sk}}, y_{\mathrm{sk}}\right)$, and $\left(x_{c}, y_{c}\right)$ signify the coordinates of the force's acting points. $\beta$ signifies slip angle, and $V_{a}$ denotes the relative wind speed which can be calculated by the following equations:

$$
\begin{aligned}
\beta & =\arctan \left(\frac{v}{u}\right), \\
V_{a} & =\sqrt{\left(u+V_{\omega} \cos \left(\beta_{\omega}-\psi\right)\right)^{2}+\left(v+V_{\omega} \sin \left(\beta_{\omega}-\psi\right)\right)^{2}}, \\
\beta_{a} & =\arctan \left[\frac{v+V_{\omega} \sin \left(\beta_{\omega}-\psi\right)}{u+V_{\omega} \cos \left(\beta_{\omega}-\psi\right)}\right],
\end{aligned}
$$

where $\beta_{a}$ signifies the relative wind direction, $V_{\omega}$ represents the absolute wind speed, and $\beta_{\omega}$ denotes the absolute wind direction.

Assumption 1. In the control process, we ignore the motion of the hovercraft's pitch and heave and set the pressure of each air chamber and the flow of the cushion fan to be constants.
Assumption 2. The same two air propellers and the same two air rudders are symmetrically mounted at the tail of the hovercraft. In addition, the air rudder provides the moment of steering and the propeller only provides the forward thrust.

Assumption 3. The model uncertainties $f_{u}, f_{v}, f_{p}$, and $f_{r}$ are continuously bounded, namely, $\left|f_{i}\right| \leq T_{i \max }$ ( $i=u, v, p, r)$ with $T_{i \max }$ being positive constants.

Assumption 4. The hovercraft's surge, sway velocities, and yaw angular velocity are bounded.

Remark 1. In practice, the velocity and yaw angular velocity of the hovercraft cannot be infinite because of the restraints of aerodynamic resistance, hydrodynamic damping term, and the ability of the actuator [35].

2.3. Problem Formulation. To expediently describe the control problem, we firstly define the reference trajectory in this paper. The hovercraft tracks that the desired path is generated by the virtual surface vessel:

$$
\left[\begin{array}{l}
\dot{x}_{d} \\
\dot{y}_{d} \\
\dot{\psi}_{d}
\end{array}\right]=\left[\begin{array}{ccc}
\cos \psi_{d} & -\sin \psi_{d} & 0 \\
\sin \psi_{d} & \cos \psi_{d} & 0 \\
0 & 0 & 1
\end{array}\right]\left[\begin{array}{c}
u_{d s \mathrm{et}} \\
v_{d s \mathrm{st}} \\
r_{d \mathrm{set}}
\end{array}\right] .
$$

Assumption 5. The virtual vessel states $x_{d}, y_{d}$, and $\psi_{d}$ and their first derivatives with respect to time $\dot{x}_{d}, \dot{y}_{d}$, and $\dot{\psi}_{d}$ are bounded.

The position tracking errors can be defined as follows:

$$
\begin{aligned}
& x_{e}=x-x_{d}, \\
& y_{e}=y-y_{d} .
\end{aligned}
$$

By utilizing (10) and (15), the derivatives of the position error with respect to time can be calculated as follows:

$$
\left[\begin{array}{c}
\dot{x}_{e} \\
\dot{y}_{e}
\end{array}\right]=\left[\begin{array}{cc}
\cos \psi & -\sin \psi \cos \phi \\
\sin \psi & \cos \psi \cos \phi
\end{array}\right]\left[\begin{array}{l}
u \\
v
\end{array}\right]-\left[\begin{array}{c}
\dot{x}_{d} \\
\dot{y}_{d}
\end{array}\right] .
$$

Subsequently, we define the velocity and yaw angular velocity tracking errors as follows:

$$
\begin{aligned}
& u_{e}=u-\alpha_{u}, \\
& v_{e}=v-\alpha_{v}, \\
& r_{e}=r-\alpha_{r},
\end{aligned}
$$

where $\alpha_{u}, \alpha_{v}$, and $\alpha_{r}$ are the virtual control laws to be designed later on, which are viewed as the desired velocities and the desired yaw angular velocity of the $u, v$, and $r$, respectively.

In terms of the equation of the hovercraft dynamics (10), the derivatives of formula (18) with respect to time can be calculated as follows: 


$$
\begin{aligned}
& \dot{u}_{e}=v r+\frac{F_{x D 0}}{m_{0}}+f_{u}+\frac{\tau_{u}}{m_{0}}-\dot{\alpha}_{u}, \\
& \dot{v}_{e}=-u r+\frac{F_{y D 0}}{m_{0}}+f_{v}-\dot{\alpha}_{v}, \\
& \dot{r}_{e}=\frac{M_{z D 0}}{J_{z 0}}+f_{r}+\frac{\tau_{r}}{J_{z 0}}-\dot{\alpha}_{r} .
\end{aligned}
$$

To avoid the dangerous navigation situation when the hovercraft sails in a narrow channel, we consider the transient performance during the position tracking error convergence. The errors can be defined to satisfy the following prescribed performance [36]:

$$
\begin{aligned}
& -\alpha_{1} \eta(t)<x_{e}<\beta_{1} \eta(t), \\
& -\alpha_{2} \eta(t)<y_{e}<\beta_{2} \eta(t),
\end{aligned}
$$

where $\eta(t)=\left(\eta_{0}-\eta_{\infty}\right) e^{-a t}+\eta_{\infty}$ is a smooth performance constraint function with parameters satisfying $\eta_{0}>\eta_{\infty}>0$, $\alpha_{i}>0, \beta_{i}>0, i=1,2$.

Assumption 6. Initial position tracking errors satisfy the strict conditions $-\alpha_{1} \eta(0)<x_{e}(0)<\beta_{1} \eta(0) \quad$ and $-\alpha_{2} \eta(0)<y_{e}(0)<\beta_{2} \eta(0)$.

The control objective in this paper can be formulated as follows.

Considering the hovercraft model (10) subject to model uncertainties and external disturbances, an adaptive neuralbased fixed-time trajectory tracking controller is designed to generate surge force $\tau_{u}$, virtual control law $\alpha_{r}$, and yaw moment $\tau_{r}$ to guarantee velocity tracking errors $u_{e}$ and $v_{e}$, and yaw angular velocity tracking error $r_{e}$ converges to the small region containing zero within the fixed time. Then, the virtual control laws $\alpha_{u}$ and $\alpha_{v}$ are reasonably designed to ensure the position tracking errors remain within the given prescribed performance bounds while the desired trajectory is being tracked by the hovercraft.

\section{Main Results}

3.1. Error Transformation. To guarantee the position tracking errors always satisfy the prescribed performance (20), the following transformations are applied:

$$
\left[\begin{array}{c}
x_{e} \\
y_{e}
\end{array}\right]=\eta(t)\left[\begin{array}{c}
S_{x}\left(\varepsilon_{x}\right) \\
S_{y}\left(\varepsilon_{y}\right)
\end{array}\right],
$$

where $S_{i}\left(\varepsilon_{i}\right), i=x, y$ are strictly monotonically increasing functions of $\varepsilon_{i}$. Select $S_{i}\left(\varepsilon_{i}\right)$ as follows:

$$
\left[\begin{array}{c}
S_{x}\left(\varepsilon_{x}\right) \\
S_{y}\left(\varepsilon_{y}\right)
\end{array}\right]=\left[\begin{array}{c}
\frac{\beta_{1} e^{\left(\varepsilon_{x}+(1 / 2) \ln \left(\alpha_{1} / \beta_{1}\right)\right)}-\alpha_{1} e^{-\left(\varepsilon_{x}+(1 / 2) \ln \left(\alpha_{1} / \beta_{1}\right)\right)}}{e^{\left(\varepsilon_{x}+(1 / 2) \ln \left(\alpha_{1} / \beta_{1}\right)\right)}+e^{-\left(\varepsilon_{x}+(1 / 2) \ln \left(\alpha_{1} / \beta_{1}\right)\right)}} \\
\frac{\beta_{2} e^{\left(\varepsilon_{y}+(1 / 2) \ln \left(\alpha_{2} / \beta_{2}\right)\right)}-\alpha_{2} e^{-\left(\varepsilon_{y}+(1 / 2) \ln \left(\alpha_{2} / \beta_{2}\right)\right)}}{e^{\left(\varepsilon_{y}+(1 / 2) \ln \left(\alpha_{2} / \beta_{2}\right)\right)}+e^{-\left(\varepsilon_{y}+(1 / 2) \ln \left(\alpha_{2} / \beta_{2}\right)\right)}}
\end{array}\right] .
$$

Since $\eta(t) \neq 0$, the transformed errors $\varepsilon_{x}$ and $\varepsilon_{y}$ can be altered as follows:

$$
\left[\begin{array}{c}
\varepsilon_{x} \\
\varepsilon_{y}
\end{array}\right]=\left[\begin{array}{l}
\frac{1}{2} \ln \left(\frac{x_{e}}{\eta} \beta_{1}+\alpha_{1} \beta_{1}\right)-\frac{1}{2} \ln \left(\alpha_{1} \beta_{1}-\frac{x_{e}}{\eta} \alpha_{1}\right) \\
\frac{1}{2} \ln \left(\frac{y_{e}}{\eta} \beta_{2}+\alpha_{2} \beta_{2}\right)-\frac{1}{2} \ln \left(\alpha_{2} \beta_{2}-\frac{y_{e}}{\eta} \alpha_{2}\right)
\end{array}\right] .
$$

Theorem 1. Regarding the position tracking errors $x_{e}$ and $y_{e}$ and the transformed errors $\varepsilon_{x}$ and $\varepsilon_{y}$, if $\varepsilon_{x}$ and $\varepsilon_{y}$ are bounded and the initial tracking errors satisfy $-\alpha_{1} \eta(0)<x_{e}(0)$ $<\beta_{1} \eta(0),-\alpha_{2} \eta(0)<y_{e}(0)<\beta_{2} \eta(0)$, then $x_{e}$ and $y_{e}$ will be ensured to stay within the prescribed performance (20) for all $t \geq 0$.

Proof. We can infer that (22) and (23) have the following properties:

$$
\begin{aligned}
& \text { (1) }-\alpha_{1}<S_{x}\left(\varepsilon_{x}\right)<\beta_{1},-\alpha_{2}<S_{y}\left(\varepsilon_{y}\right)<\beta_{2} \\
& \text { (2) } \lim _{\varepsilon_{x}} \longrightarrow-\infty S_{x}\left(\varepsilon_{x}\right)=-\alpha_{1}, \lim _{\varepsilon_{x}} \longrightarrow \infty S_{x}\left(\varepsilon_{x}\right)= \\
& \beta_{1}, \lim _{\varepsilon_{y}} \longrightarrow-\infty S_{y}\left(\varepsilon_{y}\right)=-\alpha_{2}, \lim _{\varepsilon_{y}} \longrightarrow \infty S_{y}\left(\varepsilon_{y}\right)=\beta_{2} \\
& \text { (3) } S_{x}(0)=0, S_{y}(0)=0 \\
& \text { (4) } x_{e} \longrightarrow 0 \text { when } \varepsilon_{x} \longrightarrow 0, y_{e} \longrightarrow 0 \text { when } \varepsilon_{y} \longrightarrow 0
\end{aligned}
$$

Accordingly, if $\varepsilon_{x}$ and $\varepsilon_{y}$ are not infinite and the initial tracking errors $x_{e}$ and $y_{e}$ that are within the performance limits, we can deduce that the above properties (1), (2), (3), and (4) will guarantee always that $x_{e}$ and $y_{e}$ satisfy the prespecified performance (20). And when $\varepsilon_{x}$ and $\varepsilon_{y}$ converge to a small neighbourhood around zero, $x_{e}$ and $y_{e}$ can converge to a small neighbourhood around zero as well.

3.2. Design of the Virtual Control Laws. In this subsection, we apply the Lyapunov direct method to design the desired velocities as the virtual control law of position error. Firstly, differentiate the transformed errors (23) as follows:

$$
\left[\begin{array}{c}
\dot{\varepsilon}_{x} \\
\dot{\varepsilon}_{y}
\end{array}\right]=\left[\begin{array}{cc}
\xi_{x} & 0 \\
0 & \xi_{y}
\end{array}\right]\left\{\left[\begin{array}{cc}
\cos \psi & -\sin \psi \cos \phi \\
\sin \psi & \cos \psi \cos \phi
\end{array}\right]\left[\begin{array}{l}
u \\
v
\end{array}\right]-\left[\begin{array}{c}
\dot{x}_{d}+x_{e} \frac{\dot{\eta}}{\eta} \\
\dot{y}_{d}+y_{e} \frac{\dot{\eta}}{\eta}
\end{array}\right]\right\}
$$

where

$$
\begin{aligned}
& \xi_{x}=\frac{1}{2 \eta}\left(\frac{1}{x_{e} / \eta+\alpha_{1}}-\frac{1}{x_{e} / \eta-\beta_{1}}\right), \\
& \xi_{y}=\frac{1}{2 \eta}\left(\frac{1}{y_{e} / \eta+\alpha_{2}}-\frac{1}{y_{e} / \eta-\beta_{2}}\right) .
\end{aligned}
$$

We can easily obtain that $\xi_{x} \neq 0$ and $\xi_{y} \neq 0$.

According to the fixed-time stability lemma and Lyapunov direct method, the desired velocities are designed as follows: 


$$
\begin{aligned}
{\left[\begin{array}{c}
\alpha_{u} \\
\alpha_{v}
\end{array}\right]=} & {\left[\begin{array}{cc}
\cos \psi & \sin \psi \\
\frac{-\sin \psi}{\cos \phi} & \frac{\cos \psi}{\cos \phi}
\end{array}\right] } \\
& \times\left[\begin{array}{c}
\dot{x}_{d}+x_{e} \frac{\dot{\eta}}{\eta}-\frac{1}{\xi_{x}}\left(k_{p 11} \operatorname{sign}\left(\varepsilon_{x}\right)\left|\varepsilon_{x}\right|^{p_{1}}+k_{p 12} \operatorname{sign}\left(\varepsilon_{x}\right)\left|\varepsilon_{x}\right|^{q_{1}}+k_{x} \varepsilon_{x}\right) \\
\dot{y}_{d}+y_{e} \frac{\dot{\eta}}{\eta}-\frac{1}{\xi_{y}}\left(k_{p 21} \operatorname{sign}\left(\varepsilon_{y}\right)\left|\varepsilon_{y}\right|^{p_{1}}+k_{p 22} \operatorname{sign}\left(\varepsilon_{y}\right)\left|\varepsilon_{y}\right|^{q_{1}}+k_{y} \varepsilon_{y}\right)
\end{array}\right],
\end{aligned}
$$

where $k_{x}, k_{y}, k_{p 11}, k_{p 12}, k_{p 21}, k_{p 22}, p_{1}$, and $q_{1}$ are positive constants with satisfying the inequality $0<p_{1}<1<q_{1}$.

Assumption 7. The hovercraft's roll angle $\phi$ satisfies $|\phi|<90^{\circ}$.
Remark 2. In the motion process of the hovercraft, the roll angle is impossible to reach $\pm 90^{\circ}$ because of the effect of roll restoring moment. The control task of this paper will lose its significance when the environmental forces break this balanced relationship.

By substituting (26) into (24), we have the following:

$$
\begin{aligned}
{\left[\begin{array}{c}
\dot{\varepsilon}_{x} \\
\dot{\varepsilon}_{y}
\end{array}\right]=} & {\left[\begin{array}{cc}
\xi_{x} & 0 \\
0 & \xi_{y}
\end{array}\right]\left(\left[\begin{array}{cc}
\cos \psi & -\sin \psi \cos \phi \\
\sin \psi & \cos \psi \cos \phi
\end{array}\right]\left[\begin{array}{c}
u_{e}+\alpha_{u} \\
v_{e}+\alpha_{v}
\end{array}\right]-\left[\begin{array}{c}
\dot{x}_{d}+x_{e} \frac{\dot{\eta}}{\eta} \\
\dot{y}_{d}+y_{e} \frac{\dot{\eta}}{\eta}
\end{array}\right]\right) } \\
= & \left.-\left[\begin{array}{l}
k_{p 11} \operatorname{sign}\left(\varepsilon_{x}\right)\left|\varepsilon_{x}\right|^{p_{1}}+k_{p 12} \operatorname{sign}\left(\varepsilon_{x}\right)\left|\varepsilon_{x}\right|^{q_{1}}+k_{x} \varepsilon_{x} \\
k_{p 21} \operatorname{sign}\left(\varepsilon_{y}\right)\left|\varepsilon_{y}\right|^{p_{1}}+k_{p 22} \operatorname{sign}\left(\varepsilon_{y}\right)\left|\varepsilon_{y}\right|^{q_{1}}+k_{y} \varepsilon_{y}
\end{array}\right]\right) \\
& +\left[\begin{array}{l}
u_{e} \xi_{x} \cos \psi-v_{e} \xi_{x} \sin \psi \cos \phi \\
u_{e} \xi_{y} \sin \psi+v_{e} \xi_{y} \cos \psi \cos \phi
\end{array}\right] .
\end{aligned}
$$

Consider the following candidate Lyapunov function,

$$
V_{p}=\frac{1}{2} \varepsilon_{x}^{2}+\frac{1}{2} \varepsilon_{y}^{2} \text {. }
$$

The time derivative of the Lyapunov function defined by (28) along (27) is as follows:

$$
\begin{aligned}
\dot{V}_{p}= & \varepsilon_{x} \dot{\varepsilon}_{x}+\varepsilon_{y} \dot{\varepsilon}_{y} \\
= & -k_{p 11}\left|\varepsilon_{x}\right|^{p_{1}+1}-k_{p 12}\left|\varepsilon_{x}\right|^{q_{1}+1}-k_{x} \varepsilon_{x}^{2} \\
& -k_{p 21}\left|\varepsilon_{y}\right|^{p_{1}+1}-k_{p 22}\left|\varepsilon_{y}\right|^{q_{1}+1}-k_{y} \varepsilon_{y}^{2} \\
& +\varepsilon_{x} u_{e} \xi_{x} \cos \psi-\varepsilon_{x} v_{e} \xi_{x} \sin \psi \cos \phi \\
& +\varepsilon_{y} u_{e} \xi_{y} \sin \psi+\varepsilon_{y} v_{e} \xi_{y} \cos \psi \cos \phi .
\end{aligned}
$$

The result in (29) will be applied to the stability analysis of the designed controller in Subsection 3.6.

3.3. Design of the Surge Control Law. The surge control law is designed to guarantee that the surge velocity tracking error converges to a small neighbourhood around zero within a fixed time. Consider the following candidate Lyapunov function:

$$
V_{u}=\frac{1}{2} u_{e}^{2}+\frac{1}{2} \widetilde{w}_{u}^{T} \Gamma_{u}^{-1} \widetilde{w}_{u}
$$

where $\Gamma_{u} \in R^{l_{u} \times l_{u}}$ is the positive definite diagonal matrix, $l_{u}$ is the hidden note number, and $\widetilde{w}_{u}=w_{u}^{*}-\widehat{w}_{u}$ is the estimation error of the weight value with $\widehat{w}_{u}$ representing the estimation value of the ideal weight value $w_{u}^{*}$. 
The time derivative of the Lyapunov function is defined by (30) along (19) yields the following:

$$
\begin{aligned}
\dot{V}_{u} & =u_{e} \dot{u}_{e}+\widetilde{w}_{u}^{T} \Gamma_{u}^{-1}\left(-\dot{\widehat{w}}_{u}\right) \\
& =u_{e}\left(v r+\frac{F_{x D 0}}{m_{0}}+f_{u}+\frac{\tau_{u}}{m_{0}}-\dot{\alpha}_{u}\right)+\widetilde{w}_{u}^{T} \Gamma_{u}^{-1}\left(-\dot{\widehat{w}}_{u}\right) .
\end{aligned}
$$

The ARBFNN is employed to deal with the model uncertainty $f_{u}$ of (31). According to Lemma 2, we have the following:

$$
\begin{aligned}
& f_{u}=w_{u}^{* T} h_{u}\left(z_{u}\right)+\delta_{u}\left(z_{u}\right), \\
& \hat{f}_{u}=\widehat{w}_{u}^{T} h_{u}\left(z_{u}\right),
\end{aligned}
$$

where $\hat{f}_{u}$ is the estimation value of the $f_{u}, \delta_{u}\left(z_{u}\right)$ signifies the minimum approximation error that satisfies $\left|\delta_{u}\left(z_{u}\right)\right| \leq \bar{\delta}_{u}$ with $\bar{\delta}_{u}$ being the designed positive constant, $z_{u}=[u, \dot{u}]^{T} \in \Omega_{u}$ denotes the input of the $\mathrm{NN}$, and $h_{u}\left(z_{u}\right) \in R^{l_{u}}$ is the Gaussian basis function.

According to (31), we design the surge control law as follows:

$$
\begin{aligned}
\tau_{u}= & m_{0}\left(-v r-\frac{F_{x D 0}}{m_{0}}-\hat{f}_{u}+\dot{\alpha}_{u}-k_{u} u_{e}-k_{u 1} \operatorname{sign}\left(u_{e}\right)\left|u_{e}\right|^{p_{1}}\right. \\
& \left.-k_{u 2} \operatorname{sign}\left(u_{e}\right)\left|u_{e}\right|^{q_{1}}-\varepsilon_{x} \xi_{x} \cos \psi-\varepsilon_{y} \xi_{y} \sin \psi\right),
\end{aligned}
$$

where $k_{u}>(1 / 2), k_{u 1}$, and $k_{u 2}$ are positive constants and the updated law of the NN weight is designed as follows:

$$
-\dot{\widehat{w}}_{u}=-u_{e} \Gamma_{u} h_{u}\left(z_{u}\right)+\sigma_{u} \Gamma_{u} \widehat{w}_{u} .
$$

By substituting (32) and (33) into (31), we can obtain the derivative of the Lyapunov function $V_{u}$ as follows:

$$
\begin{aligned}
\dot{V}_{u}= & u_{e}\left(v r+\frac{F_{x D 0}}{m_{0}}+f_{u}+\frac{\tau_{u}}{m_{0}}-\dot{\alpha}_{u}\right)+\widetilde{w}_{u}^{T} \Gamma_{u}^{-1}\left(-\dot{\widehat{w}}_{u}\right) \\
= & u_{e}\left(f_{u}-\widehat{f}_{u}\right)-k_{u} u_{e}^{2}-k_{u 1}\left|u_{e}\right|^{p_{1}+1} \\
& -k_{u 2}\left|u_{e}\right|^{q_{1}+1}-u_{e} \varepsilon_{x} \xi_{x} \cos \psi \\
& -u_{e} \varepsilon_{y} \xi_{y} \sin \psi+\widetilde{w}_{u}^{T} \Gamma_{u}^{-1}\left(-u_{e} \Gamma_{u} h_{u}(z)+\sigma_{u} \widehat{w}_{u}\right) \\
= & -k_{u} u_{e}^{2}-k_{u 1}\left|u_{e}\right|^{p_{1}+1}-k_{u 2}\left|u_{e}\right|^{q_{1}+1}-u_{e} \varepsilon_{x} \xi_{x} \cos \psi \\
& -u_{e} \varepsilon_{y} \xi_{y} \sin \psi+\widetilde{w}_{u}^{T} \sigma_{u} \widehat{w}_{u}+u_{e} \delta_{u}\left(z_{u}\right) \\
\leq & -\left(k_{u}-\frac{1}{2}\right) u_{e}^{2}-k_{u 1}\left|u_{e}\right|^{p_{1}+1}-k_{u 2}\left|u_{e}\right|^{q_{1}+1}-u_{e} \varepsilon_{x} \xi_{x} \cos \psi \\
& -u_{e} \varepsilon_{y} \xi_{y} \sin \psi+\widetilde{w}_{u}^{T} \sigma_{u} \widehat{w}_{u}+\frac{1}{2} \delta_{u}^{2} .
\end{aligned}
$$

Inequality (35) will be used to analyse the stability of the controlled system in Subsection 3.6.

3.4. Design of the Desired Angular Velocity in Yaw. The hovercraft is a kind of typical underactuated surface vessel because its lateral axis is not directly actuated. Thus, the virtual control law is designed to stabilize the sway velocity tracking error. Consider the following candidate Lyapunov function:

$$
V_{v}=\frac{1}{2} v_{e}^{2}+\frac{1}{2} \widetilde{w}_{v}^{T} \Gamma_{v}^{-1} \widetilde{w}_{v}
$$

where $\Gamma_{v} \in R^{l_{v} \times l_{v}}$ signifies the design positive definite diagonal matrix with $l_{v}$ being the hidden note number, $\widetilde{w}_{v}=$ $w_{v}^{*}-\widehat{w}_{v}$ denotes the estimation error of the weight value, and the ideal weight value $w_{v}^{*}$ is estimated by the $\widehat{w}_{v}$.

The derivative of the Lyapunov function $V_{v}$ with respect to time is given by the following:

$$
\begin{aligned}
\dot{V}_{v} & =v_{e} \dot{v}_{e}+\widetilde{w}_{v}^{T} \Gamma_{v}^{-1}\left(-\dot{\widehat{w}}_{v}\right) \\
& =v_{e}\left(-u r+\frac{F_{y D 0}}{m_{0}}+f_{v}-\dot{\alpha}_{v}\right)+\widetilde{w}_{v}^{T} \Gamma_{v}^{-1}\left(-\dot{\widehat{w}}_{v}\right) .
\end{aligned}
$$

The model uncertainty $f_{v}$ of (37) is handled by applying the ARBFNN. According to Lemma 2, we can obtain the following:

$$
\begin{aligned}
& f_{v}=w_{v}^{* T} h_{v}\left(z_{v}\right)+\delta_{v}\left(z_{v}\right), \\
& \widehat{f}_{v}=\widehat{w}_{v}^{T} h_{v}\left(z_{v}\right),
\end{aligned}
$$

where $\widehat{f}_{v}$ is the estimation value of $f_{v}$, the minimum approximation error is represented by $\delta_{v}\left(z_{v}\right)$ and satisfies $\left|\delta_{v}\left(z_{v}\right)\right| \leq \bar{\delta}_{v}$ with $\bar{\delta}_{v}$ being the design positive constant, $z_{v}=$ $[v, \dot{v}]^{T} \in \Omega_{v}$ denotes the input of the $\mathrm{NN}$, and $h_{v}\left(z_{v}\right) \in R^{l_{v}}$ is the Gaussian basis function.

According to (37), the design desired yaw angular velocity as a stability function:

$$
\begin{aligned}
\alpha_{r}= & \frac{1}{u}\left(\frac{F_{y D 0}}{m_{0}}-\dot{\alpha}_{v}+\hat{f}_{v}+k_{v} v_{e}+k_{v 1} \operatorname{sign}\left(v_{e}\right)\left|v_{e}\right|^{p_{1}}+k_{v 2} \operatorname{sign}\left(v_{e}\right)\left|v_{e}\right|^{q_{1}}\right. \\
& \left.-\varepsilon_{x} \xi_{x} \sin \psi \cos \phi+\varepsilon_{y} \xi_{y} \cos \psi \cos \phi\right),
\end{aligned}
$$

where $k_{v}>(1 / 2), k_{v 1}$, and $k_{v 2}$ are positive constants and the updated law of the NN weight is designed as follows:

$$
-\dot{\widehat{w}}_{v}=-v_{e} \Gamma_{v} h_{v}(z)+\sigma_{v} \Gamma_{v} \widehat{w}_{v}
$$

Remark 3. In the practical motion control of the hovercraft, the pitch angle is always set to the positive value. Only in special cases, the negative pitch angle can be set, such as when the hovercraft enters and leaves the mother ship. Accordingly, the surge velocity is always set to $u>0$ in the motion control process of the hovercraft.

By substituting (38) and (39) into (40), we can obtain the derivative of the Lyapunov function $V_{v}$ as follows: 


$$
\begin{aligned}
\dot{V}_{v}= & v_{e}\left(-u r+\frac{F_{y D 0}}{m_{0}}+f_{v}-\dot{\alpha}_{v}\right)+\widetilde{w}_{v}^{T} \Gamma_{v}^{-1}\left(-\dot{\hat{w}}_{v}\right) \\
= & v_{e}\left(-k_{v} v_{e}-k_{v 1} \operatorname{sign}\left(v_{e}\right)\left|v_{e}\right|^{p_{1}}-k_{v 2} \operatorname{sign}\left(v_{e}\right)\left|v_{e}\right|^{q_{1}}+f_{v}-\widehat{f}_{v}\right) \\
& +\widetilde{w}_{v}^{T} \Gamma_{v}^{-1}\left(-\dot{\hat{w}}_{v}\right)+\varepsilon_{x} v_{e} \xi_{x} \sin \psi \cos \phi-\varepsilon_{y} v_{e} \xi_{y} \cos \psi \cos \phi \\
= & -u v_{e} r_{e}+v_{e} \delta_{v}\left(z_{v}\right)-k_{v} v_{e}^{2}-k_{v 1}\left|v_{e}\right|^{p_{1}+1}-k_{v 2}\left|v_{e}\right|^{q_{1}+1}+\sigma_{v} \widetilde{w}_{v}^{T} \widehat{w}_{v} \\
\leq & -\left(k_{v}-\frac{1}{2}\right) v_{e}^{2}-k_{v 1}\left|v_{e}\right|^{p_{1}+1}-k_{v 2}\left|v_{e}\right|^{q_{1}+1}+\sigma_{v} \widetilde{w}_{v}^{T} \widehat{w}_{v} \\
& +\frac{1}{2} \bar{\delta}_{v}^{2}-u v_{e} r_{e}+\varepsilon_{x} v_{e} \xi_{x} \sin \psi \cos \phi-\varepsilon_{y} v_{e} \xi_{y} \cos \psi \cos \phi .
\end{aligned}
$$

Inequality (41) will be used to perform the stability analysis of the nonlinear system in Subsection 3.6.

3.5. Design of the Yaw Control Law. Subsequently, we design the yaw control law $\tau_{r}$ to guarantee that the tracking error of angular velocity in the yaw converges to a small region around zero within a fixed time. Select the Lyapunov candidate function as follows:

$$
V_{r}=\frac{1}{2} r_{e}^{2}+\frac{1}{2} \widetilde{w}_{r}^{T} \Gamma_{r}^{-1} \widetilde{w}_{r}
$$

where $\Gamma_{r} \in R^{l_{r} \times l_{r}}$ is the designed positive definite diagonal matrix, $l_{r}$ is the hidden note number, and the estimation error of the weight value is represented by $\widetilde{w}_{r}=w_{r}^{*}-\widehat{w}_{r}$ with $\widehat{w}_{r}$ denoting the estimation value of the ideal weight value $w_{r}^{*}$.

The time derivative of the Lyapunov function defined by (42) along (19) is calculated as follows:

$$
\begin{aligned}
\dot{V}_{r} & =r_{e} \dot{r}_{e}+\widetilde{w}_{r}^{T} \Gamma_{r}^{-1}\left(-\dot{\hat{w}}_{r}\right) \\
& =r_{e}\left(\frac{M_{z D 0}}{J_{z 0}}+f_{r}+\frac{\tau_{r}}{J_{z 0}}-\dot{\alpha}_{r}\right)+\widetilde{w}_{r}^{T} \Gamma_{r}^{-1}\left(-\dot{\widehat{w}}_{r}\right) .
\end{aligned}
$$

The model uncertainty $f_{r}$ of (43) is tackled by applying the ARBFNN. Accordingly, in terms of Lemma 2, we have the following:

$$
\begin{aligned}
& f_{r}=w_{r}^{* T} h_{r}\left(z_{r}\right)+\delta_{r}\left(z_{r}\right), \\
& \hat{f}_{r}=\widehat{w}_{r}^{T} h_{r}\left(z_{r}\right),
\end{aligned}
$$

where $\hat{f}_{r}$ is the estimation value of the $f_{r}$, the minimum approximation error is signified by $\delta_{r}\left(z_{r}\right)$ and satisfies $\left|\delta_{r}\left(z_{r}\right)\right| \leq \bar{\delta}_{r}$ with $\bar{\delta}_{r}$ being the designed positive constant, $z_{r}=[r, \dot{r}]^{T} \in \Omega_{r}$ denotes the input of the $\mathrm{NN}$, and $h_{r}\left(z_{r}\right) \in R^{l_{r}}$ is the Gaussian basis function.

According to (43), design the yaw control law as follows:

$$
\tau_{r}=J_{z 0}\left(\dot{\alpha}_{r}-\frac{M_{z D 0}}{J_{z 0}}-\hat{f}_{r}-k_{r} r_{e}-k_{r 1} \operatorname{sign}\left(r_{e}\right)\left|r_{e}\right|^{p_{1}}-k_{r 2} \operatorname{sign}\left(r_{e}\right)\left|r_{e}\right|^{q_{1}}+u v_{e}\right),
$$

where $k_{r}>(1 / 2), k_{r 1}$ and $k_{r 2}$ are positive constants, and the updated law of the NN weight is selected as follows:

$$
-\dot{\widehat{w}}_{r}=-r_{e} \Gamma_{r} h_{r}\left(z_{r}\right)+\sigma_{r} \Gamma_{r} \widehat{w}_{r} .
$$

Then, substituting (44) and (45) into (43), the derivative of the Lyapunov function is expressed as follows:

$$
\begin{aligned}
\dot{V}_{r}= & r_{e}\left(f_{r}-\widehat{f}_{r}-k_{r} r_{e}-k_{r 1} \operatorname{sign}\left(r_{e}\right)\left|r_{e}\right|^{p_{1}}\right. \\
& \left.-k_{r 2} \operatorname{sign}\left(r_{e}\right)\left|r_{e}\right|^{q_{1}}+u v_{e}\right) \\
& +\widetilde{w}_{r}^{T} \Gamma_{r}^{-1}\left(-r_{e} \Gamma_{r} h_{r}\left(z_{r}\right)+\sigma_{r} \Gamma_{r} \widehat{w}_{r}\right) \\
= & r_{e}\left(\widetilde{w}_{r}^{T} h_{r}\left(z_{r}\right)+\delta_{r}\left(z_{r}\right)\right)-k_{r 1}\left|r_{e}\right|^{p_{1}+1}-k_{r 2}\left|r_{e}\right|^{q_{1}+1}+u v_{e} r_{e} \\
& +\widetilde{w}_{r}^{T} \Gamma_{r}^{-1}\left(-r_{e} \Gamma_{r} h_{r}\left(z_{r}\right)+\sigma_{r} \Gamma_{r} \widehat{w}_{r}\right)-k_{r} r_{e}^{2} \\
= & r_{e} \delta_{r}\left(z_{r}\right)-k_{r 1}\left|r_{e}\right|^{p_{1}+1}-k_{r 2}\left|r_{e}\right|^{q_{1}+1} \\
& +u v_{e} r_{e}+\sigma_{r} \widetilde{w}_{r}^{T} \widehat{w}-k_{r} r_{e}^{2} \\
\leq & -\left(k_{r}-\frac{1}{2}\right) r_{e}^{2}-k_{r 1}\left|r_{e}\right|^{p_{1}+1}-k_{r 2}\left|r_{e}\right|^{q_{1}+1} \\
& +u v_{e} r_{e}+\sigma_{r} \widetilde{w}_{r}^{T} \widehat{w}+\frac{1}{2} \bar{\delta}_{r}^{2} .
\end{aligned}
$$

The result in (47) will be applied to the stability analysis of the control system in the next subsection.

\subsection{Stability Analysis}

Theorem 2. Consider the hovercraft trajectory tracking nonlinear system (10) under model uncertainty and external environment disturbance, and suppose that Assumptions 1-7 are satisfied. If the desired velocities $\alpha_{u}$ and $\alpha_{v}$ and desired angular velocity $\alpha_{r}$ are calculated by (26) and (39), the adaptive fixed-time controllers $\tau_{u}$ and $\tau_{r}$ are obtained by (33) and (45), the model uncertainties and external disturbances are approximated by the ARBFNNs (32), (38), and (44), and the initial condition satisfies $-\alpha_{1} \eta(0)<x_{e}(0)<\beta_{1} \eta(0)$ and $-\alpha_{2} \eta(0)<y_{e}(0)<\beta_{2} \eta(0)$, then the position tracking errors can be guaranteed to remain within the given prescribed performance bounds (20); all tracking errors can converge to a small region containing zero within the fixed time and are uniformly ultimately bounded.

Proof. The proof process is divided into two steps. Firstly, we indicate that all error signals are uniformly ultimately bounded. Secondly, verify that all tracking errors will converge uniformly to a small neighbourhood around zero within a fixed time. 
Step 1. Consider the Lyapunov candidate function of the system:

$$
\begin{aligned}
V= & V_{p}+V_{u}+V_{v}+V_{r} \\
= & \frac{1}{2} \varepsilon_{x}^{2}+\frac{1}{2} \varepsilon_{y}^{2}+\frac{1}{2} u_{e}^{2}+\frac{1}{2} \widetilde{w}_{u}^{T} \Gamma_{u}^{-1} \widetilde{w}_{u} \\
& +\frac{1}{2} v_{e}^{2}+\frac{1}{2} \widetilde{w}_{v}^{T} \Gamma_{v}^{-1} \widetilde{w}_{v}+\frac{1}{2} r_{e}^{2}+\frac{1}{2} \widetilde{w}_{r}^{T} \Gamma_{r}^{-1} \widetilde{w}_{r} .
\end{aligned}
$$

With the help of (29), (35), (41), and (47), the time derivative of (48) satisfies the following:

$$
\begin{aligned}
& \dot{V} \leq-k_{p 11}\left|\varepsilon_{x}\right|^{p_{1}+1}-k_{p 12}\left|\varepsilon_{x}\right|^{q_{1}+1}-k_{x} \varepsilon_{x}^{2}-k_{p 21}\left|\varepsilon_{y}\right|^{p_{1}+1}-k_{p 22}\left|\varepsilon_{y}\right|^{q_{1}+1}-k_{y} \varepsilon_{y}^{2} \\
& +\varepsilon_{x} u_{e} \xi_{x} \cos \psi-\varepsilon_{x} v_{e} \xi_{x} \sin \psi \cos \phi+\varepsilon_{y} u_{e} \xi_{y} \sin \psi+\varepsilon_{y} v_{e} \xi_{y} \cos \psi \cos \phi \\
& -\left(k_{u}-\frac{1}{2}\right) u_{e}^{2}-k_{u 1}\left|u_{e}\right|^{p_{1}+1}-k_{u 2}\left|u_{e}\right|^{q_{1}+1}-u_{e} \varepsilon_{x} \xi_{x} \cos \psi-u_{e} \varepsilon_{y} \xi_{y} \sin \psi \\
& +\widetilde{w}_{u}^{T} \sigma_{u} \widehat{w}_{u}+\frac{1}{2} \bar{\delta}_{u}^{2}-\left(k_{v}-\frac{1}{2}\right) v_{e}^{2}-k_{v 1}\left|v_{e}\right|^{p_{1}+1}-k_{v 2}\left|v_{e}\right|^{q_{1}+1}+\sigma_{v} \widetilde{w}_{v}^{T} \widehat{w}_{v} \\
& +\frac{1}{2} \bar{\delta}_{v}^{2}-u v_{e} r_{e}+\varepsilon_{x} v_{e} \xi_{x} \sin \psi \cos \phi-\varepsilon_{y} v_{e} \xi_{y} \cos \psi \cos \phi \\
& -\left(k_{r}-\frac{1}{2}\right) r_{e}^{2}-k_{r 1}\left|r_{e}\right|^{p_{1}+1}-k_{r 2}\left|r_{e}\right|^{q_{1}+1}+u v_{e} r_{e}+\sigma_{r} \widetilde{w}_{r}^{T} \widehat{w}+\frac{1}{2} \bar{\delta}_{r}^{2} \\
& =-k_{p 11}\left|\varepsilon_{x}\right|^{p_{1}+1}-k_{p 12}\left|\varepsilon_{x}\right|^{q_{1}+1}-k_{x} \varepsilon_{x}^{2}-k_{p 21}\left|\varepsilon_{y}\right|^{p_{1}+1}-k_{p 22}\left|\varepsilon_{y}\right|^{q_{1}+1}-k_{y} \varepsilon_{y}^{2} \\
& -\left(k_{u}-\frac{1}{2}\right) u_{e}^{2}-k_{u 1}\left|u_{e}\right|^{p_{1}+1}-k_{u 2}\left|u_{e}\right|^{q_{1}+1}-\left(k_{v}-\frac{1}{2}\right) v_{e}^{2}-k_{v 1}\left|v_{e}\right|^{p_{1}+1} \\
& -k_{v 2}\left|v_{e}\right|^{q_{1}+1}-\left(k_{r}-\frac{1}{2}\right) r_{e}^{2}-k_{r 1}\left|r_{e}\right|^{p_{1}+1}-k_{r 2}\left|r_{e}\right|^{q_{1}+1}-\sigma_{u} \widetilde{w}_{u}^{T} \widetilde{w}_{u}+\frac{1}{2} \bar{\delta}_{u}^{2} \\
& -\sigma_{v} \widetilde{w}_{v}^{T} \widetilde{w}_{v}-\sigma_{r} \widetilde{w}_{r}^{T} \widetilde{w}_{r}+\sigma_{u} \widetilde{w}_{u}^{T} w_{u}^{*}+\sigma_{v} \widetilde{w}_{v}^{T} w_{v}^{*}+\sigma_{r} \widetilde{w}_{r}^{T} w_{r}^{*}+\frac{1}{2} \bar{\delta}_{v}^{2}+\frac{1}{2} \bar{\delta}_{r}^{2} .
\end{aligned}
$$


In the light of Young's inequality, the following inequalities can be obtained:

$$
\begin{aligned}
& \sigma_{u} \widetilde{w}_{u}^{T} w_{u}^{*} \leq \frac{\sigma_{u}}{2} \widetilde{w}_{u}^{T} \widetilde{w}_{u}+\frac{\sigma_{u}}{2} w_{u}^{* T} w_{u}^{*}, \\
& \sigma_{v} \widetilde{w}_{v}^{T} w_{v}^{*} \leq \frac{\sigma_{v}}{2} \widetilde{w}_{v}^{T} \widetilde{w}_{v}+\frac{\sigma_{v}}{2} w_{v}^{* T} w_{v}^{*}, \\
& \sigma_{r} \widetilde{w}_{r}^{T} w_{r}^{*} \leq \frac{\sigma_{r}}{2} \widetilde{w}_{r}^{T} \widetilde{w}_{r}+\frac{\sigma_{r}}{2} w_{r}^{* T} w_{r}^{*} .
\end{aligned}
$$

Thus, we can rewrite (49) as follows:

$$
\begin{aligned}
\dot{V} \leq & -k_{p 11}\left|\varepsilon_{x}\right|^{p_{1}+1}-k_{p 12}\left|\varepsilon_{x}\right|^{q_{1}+1}-k_{x} \varepsilon_{x}^{2}-k_{p 21}\left|\varepsilon_{y}\right|^{p_{1}+1}-k_{p 22}\left|\varepsilon_{y}\right|^{q_{1}+1}-k_{y} \varepsilon_{y}^{2}-\left(k_{u}-\frac{1}{2}\right) u_{e}^{2} \\
& -k_{u 1}\left|u_{e}\right|^{p_{1}+1}-k_{u 2}\left|u_{e}\right|^{q_{1}+1}-\frac{\sigma_{u}}{2} \widetilde{w}_{u}^{T} \widetilde{w}_{u}-\left(k_{v}-\frac{1}{2}\right) v_{e}^{2}-k_{v 1}\left|v_{e}\right|^{p_{1}+1}-k_{v 2}\left|v_{e}\right|^{q_{1}+1}-\frac{\sigma_{v}}{2} \widetilde{w}_{v}^{T} \widetilde{w}_{v} \\
& -\left(k_{r}-\frac{1}{2}\right) r_{e}^{2}-k_{r 1}\left|r_{e}\right|^{p_{1}+1}-k_{r 2}\left|r_{e}\right|^{q_{1}+1}-\frac{\sigma_{r}}{2} \widetilde{w}_{r}^{T} \widetilde{w}_{r}+\frac{\sigma_{u}}{2} w_{u}^{* T} w_{u}^{*}+\frac{\sigma_{v}}{2} w_{v}^{* T} w_{v}^{*}+\frac{\sigma_{r}}{2} w_{r}^{* T} w_{r}^{*} \\
& +\frac{1}{2} \bar{\delta}_{v}^{2}+\frac{1}{2} \bar{\delta}_{u}^{2}+\frac{1}{2} \bar{\delta}_{r}^{2} \\
\leq & -k_{x} \varepsilon_{x}^{2}-k_{y} \varepsilon_{y}^{2}-\left(k_{u}-\frac{1}{2}\right) u_{e}^{2}-\frac{\sigma_{u}}{2} \widetilde{w}_{u}^{T} \widetilde{w}_{u} \\
& -\left(k_{v}-\frac{1}{2}\right) v_{e}^{2}-\frac{\sigma_{v}}{2} \widetilde{w}_{v}^{T} \widetilde{w}_{v}-\left(k_{r}-\frac{1}{2}\right) r_{e}^{2}-\frac{\sigma_{r}}{2} \widetilde{w}_{r}^{T} \widetilde{w}_{r} \\
& +\frac{\sigma_{u}}{2} w_{u}^{* T} w_{u}^{*}+\frac{\sigma_{v}}{2} w_{v}^{* T} w_{v}^{*}+\frac{\sigma_{r}}{2} w_{r}^{* T} w_{r}^{*}+\frac{1}{2} \bar{\delta}_{v}^{2}+\frac{1}{2} \bar{\delta}_{u}^{2}+\frac{1}{2} \bar{\delta}_{r}^{2} \\
\leq & -2 \rho V+C_{1},
\end{aligned}
$$

where

$$
\begin{aligned}
\rho & =\min \left\{k_{x}, k_{y},\left(k_{u}-\frac{1}{2}\right),\left(k_{v}-\frac{1}{2}\right),\left(k_{r}-\frac{1}{2}\right), \frac{\sigma_{u}}{2} \lambda_{\min }\left(\Gamma_{u}\right), \frac{\sigma_{v}}{2} \lambda_{\min }\left(\Gamma_{v}\right), \frac{\sigma_{r}}{2} \lambda_{\min }\left(\Gamma_{r}\right)\right\}, \\
C_{1} & =\frac{\sigma_{u}}{2} w_{u}^{* T} w_{u}^{*}+\frac{\sigma_{v}}{2} w_{v}^{* T} w_{v}^{*}+\frac{\sigma_{r}}{2} w_{r}^{* T} w_{r}^{*}+\frac{1}{2} \bar{\delta}_{v}^{2}+\frac{1}{2} \bar{\delta}_{u}^{2}+\frac{1}{2} \bar{\delta}_{r}^{2} .
\end{aligned}
$$

By integrating both sides of (51), the following inequality is obtained:

$$
0 \leq V \leq\left(V(0)-\frac{C_{1}}{2 \rho}\right) e^{-2 \rho t}+\frac{C_{1}}{2 \rho} .
$$

Therefore, it is clear that $V$ is bounded, which implies that the tracking errors are uniformly ultimately bounded. Thus, in terms of Theorem 1, we can deduce that the position tracking errors $x_{e}$ and $y_{e}$ always satisfy their prescribed performance limits (20). Furthermore, we can reasonably 
suppose that there is always a positive constant $\Delta_{M}$ such that $\left\|\widetilde{w}_{i}\right\| \leq \Delta_{M}, i=u, v, r$.
Step 2. For indicating that the tracking errors of the controlled system are uniformly fixed-time stable, we alter inequality (51) as follows:

$$
\begin{aligned}
& \dot{V} \leq-k_{p 11} 2^{\left(p_{1}+1 / 2\right)}\left(\frac{1}{2} \varepsilon_{x}^{2}\right)^{\left(p_{1}+1 / 2\right)}-k_{p 21} 2^{\left(p_{1}+1 / 2\right)}\left(\frac{1}{2} \varepsilon_{y}^{2}\right)^{\left(p_{1}+1 / 2\right)}-k_{u 1} 2^{\left(p_{1}+1 / 2\right)}\left(\frac{1}{2} u_{e}^{2}\right)^{\left(p_{1}+1 / 2\right)} \\
& -k_{v 1} 2^{\left(p_{1}+1 / 2\right)}\left(\frac{1}{2} v_{e}^{2}\right)^{\left(p_{1}+1 / 2\right)}-k_{r 1} 2^{\left(p_{1}+1 / 2\right)}\left(\frac{1}{2} r_{e}^{2}\right)^{\left(p_{1}+1 / 2\right)}-k_{p 12} 2^{\left(q_{1}+1 / 2\right)}\left(\frac{1}{2} \varepsilon_{x}^{2}\right)^{\left(q_{1}+1 / 2\right)} \\
& -k_{p 22} 2^{\left(q_{1}+1 / 2\right)}\left(\frac{1}{2} \varepsilon_{y}^{2}\right)^{\left(q_{1}+1 / 2\right)}-k_{u 2} 2^{\left(q_{1}+1 / 2\right)}\left(\frac{1}{2} u_{e}^{2}\right)^{\left(q_{1}+1 / 2\right)}-k_{v 2} 2^{\left(q_{1}+1 / 2\right)}\left(\frac{1}{2} v_{e}^{2}\right)^{\left(q_{1}+1 / 2\right)} \\
& -k_{r 2} 2^{\left(q_{1}+1 / 2\right)}\left(\frac{1}{2} r_{e}^{2}\right)^{\left(q_{1}+1 / 2\right)}+\frac{\sigma_{u}}{2} w_{u}^{* T} w_{u}^{*}+\frac{\sigma_{v}}{2} w_{v}^{* T} w_{v}^{*}+\frac{\sigma_{r}}{2} w_{r}^{* T} w_{r}^{*} \\
& -\left(\sigma_{u} \lambda_{\min }\left(\Gamma_{u}\right)\right)^{\left(p_{1}+1 / 2\right)}\left(\frac{1}{2} \widetilde{w}_{u}^{T} \Gamma_{u}^{-1} \widetilde{w}_{u}\right)^{\left(p_{1}+1 / 2\right)}+\left(\frac{\sigma_{u}}{2} \lambda_{\min }\left(\Gamma_{u}\right) \widetilde{w}_{u}^{T} \Gamma_{u}^{-1} \widetilde{w}_{u}\right)^{\left(p_{1}+1 / 2\right)} \\
& -\left(\sigma_{v} \lambda_{\min }\left(\Gamma_{v}\right)\right)^{\left(p_{1}+1 / 2\right)}\left(\frac{1}{2} \widetilde{w}_{v}^{T} \Gamma_{v}^{-1} \widetilde{w}_{v}\right)^{\left(p_{1}+1 / 2\right)}+\left(\frac{\sigma_{v}}{2} \lambda_{\min }\left(\Gamma_{v}\right) \widetilde{w}_{v}^{T} \Gamma_{v}^{-1} \widetilde{w}_{v}\right)^{\left(p_{1}+1 / 2\right)} \\
& -\left(\sigma_{r} \lambda_{\min }\left(\Gamma_{r}\right)\right)^{\left(p_{1}+1 / 2\right)}\left(\frac{1}{2} \widetilde{w}_{r}^{T} \Gamma_{r}^{-1} \widetilde{w}_{r}\right)^{\left(p_{1}+1 / 2\right)}+\left(\frac{\sigma_{r}}{2} \lambda_{\min }\left(\Gamma_{r}\right) \widetilde{w}_{r}^{T} \Gamma_{r}^{-1} \widetilde{w}_{r}\right)^{\left(p_{1}+1 / 2\right)} \\
& -\frac{\sigma_{u}}{2} \lambda_{\min }\left(\Gamma_{u}\right) \widetilde{w}_{u}^{T} \Gamma_{u}^{-1} \widetilde{w}_{u}-\frac{\sigma_{v}}{2} \lambda_{\min }\left(\Gamma_{v}\right) \widetilde{w}_{v}^{T} \Gamma_{v}^{-1} \widetilde{w}_{v}-\frac{\sigma_{r}}{2} \lambda_{\min }\left(\Gamma_{r}\right) \widetilde{w}_{r}^{T} \Gamma_{r}^{-1} \widetilde{w}_{r} \\
& -\left(\sigma_{u} \lambda_{\min }\left(\Gamma_{u}\right)\right)^{\left(q_{1}+1 / 2\right)}\left(\frac{1}{2} \widetilde{w}_{u}^{T} \Gamma_{u}^{-1} \widetilde{w}_{u}\right)^{\left(q_{1}+1 / 2\right)}+\left(\frac{\sigma_{u}}{2} \lambda_{\min }\left(\Gamma_{u}\right) \widetilde{w}_{u}^{T} \Gamma_{u}^{-1} \widetilde{w}_{u}\right)^{\left(q_{1}+1 / 2\right)} \\
& -\left(\sigma_{v} \lambda_{\min }\left(\Gamma_{v}\right)\right)^{\left(q_{1}+1 / 2\right)}\left(\frac{1}{2} \widetilde{w}_{v}^{T} \Gamma_{v}^{-1} \widetilde{w}_{v}\right)^{\left(q_{1}+1 / 2\right)}+\left(\frac{\sigma_{v}}{2} \lambda_{\min }\left(\Gamma_{v}\right) \widetilde{w}_{v}^{T} \Gamma_{v}^{-1} \widetilde{w}_{v}\right)^{\left(q_{1}+1 / 2\right)} \\
& -\left(\sigma_{r} \lambda_{\min }\left(\Gamma_{r}\right)\right)^{\left(q_{1}+1 / 2\right)}\left(\frac{1}{2} \widetilde{w}_{r}^{T} \Gamma_{r}^{-1} \widetilde{w}_{r}\right)^{\left(q_{1}+1 / 2\right)}+\left(\frac{\sigma_{r}}{2} \lambda_{\min }\left(\Gamma_{r}\right) \widetilde{w}_{r}^{T} \Gamma_{r}^{-1} \widetilde{w}_{r}\right)^{\left(q_{1}+1 / 2\right)} \\
& +\frac{1}{2} \bar{\delta}_{u}^{2}+\frac{1}{2} \bar{\delta}_{v}^{2}+\frac{1}{2} \bar{\delta}_{r}^{2}
\end{aligned}
$$

With the fact that for all real numbers $x \geq 0$, we can obtain the following inequality:

$$
x^{\left(p_{1}+1 / 2\right)}-x \leq\left(\frac{p_{1}+1}{2}\right)^{\left(p_{1}+1 / 1-p_{1}\right)}-\left(\frac{p_{1}+1}{2}\right)^{\left(2 / 1-p_{1}\right)}=\vartheta_{0} .
$$

According to Lemma 1 and (55), inequality (54) can be expressed as follows:

$$
\dot{V} \leq-\rho_{1} V^{\left(p_{1}+1 / 2\right)}-8^{\left(1-q_{1} / 2\right)} \rho_{2} V^{\left(q_{1}+1 / 2\right)}+C_{2},
$$

where 


$$
\begin{aligned}
& \rho_{1}=\min \left\{\begin{array}{l}
k_{p 11} 2^{\left(p_{1}+1 / 2\right)}, k_{p 21} 2^{\left(p_{1}+1 / 2\right)}, k_{u 1} 2^{\left(p_{1}+1 / 2\right)},\left(\sigma_{r} \lambda_{\min }\left(\Gamma_{r}\right)\right)^{\left(p_{1}+1 / 2\right)}, \\
k_{v 1} 2^{\left(p_{1}+1 / 2\right)}, k_{r 1} 2^{\left(p_{1}+1 / 2\right)},\left(\sigma_{u} \lambda_{\min }\left(\Gamma_{u}\right)\right)^{\left(p_{1}+1 / 2\right)},\left(\sigma_{v} \lambda_{\min }\left(\Gamma_{v}\right)\right)^{\left(p_{1}+1 / 2\right)},
\end{array}\right. \\
& \rho_{2}=\min \left\{\begin{array}{l}
k_{p 12} 2^{\left(q_{1}+1 / 2\right)}, k_{p 22} 2^{\left(q_{1}+1 / 2\right)},\left(\sigma_{r} \lambda_{\min }\left(\Gamma_{r}\right)\right)^{\left(q_{1}+1 / 2\right)}, \\
k_{u 2} 2^{\left(q_{1}+1 / 2\right)}, k_{v 2} 2^{\left(q_{1}+1 / 2\right)},\left(\sigma_{u} \lambda_{\min }\left(\Gamma_{u}\right)\right)^{\left(q_{1}+1 / 2\right)}, \\
k_{r 2} 2^{\left(q_{1}+1 / 2\right)},\left(\sigma_{v} \lambda_{\min }\left(\Gamma_{v}\right)\right)^{\left(q_{1}+1 / 2\right)},
\end{array}\right. \\
& \vartheta_{1}=\left(\frac{\sigma_{u}}{2} \lambda_{\min }\left(\Gamma_{u}\right) \lambda_{\max }\left(\Gamma_{u}^{-1}\right)\right)^{\left(q_{1}+1 / 2\right)}+\left(\frac{\sigma_{v}}{2} \lambda_{\min }\left(\Gamma_{v}\right) \lambda_{\max }\left(\Gamma_{v}^{-1}\right)\right)^{\left(q_{1}+1 / 2\right)}+\left(\frac{\sigma_{r}}{2} \lambda_{\min }\left(\Gamma_{r}\right) \lambda_{\max }\left(\Gamma_{r}^{-1}\right)\right)^{\left(q_{1}+1 / 2\right)}, \\
& C_{2}=\frac{1}{2} \bar{\delta}_{v}^{2}+\frac{1}{2} \bar{\delta}_{u}^{2}+\frac{1}{2} \bar{\delta}_{r}^{2}+3 \vartheta_{0}+\vartheta_{1} \Delta_{M}^{q_{1}+1}+\frac{\sigma_{u}}{2} w_{u}^{* T} w_{u}^{*}+\frac{\sigma_{v}}{2} w_{v}^{* T} w_{v}^{*}+\frac{\sigma_{r}}{2} w_{r}^{* T} w_{r}^{*} .
\end{aligned}
$$

In terms of Lemma 4, all tracking errors can converge to the residual set $D_{\text {total }}=D_{\text {total_1 }} \cup D_{\text {total_2 }}$ within a fixed time $T_{\text {total }}$. Then, $D_{\text {total }}$ and $T_{\text {total }}$ can be formulated as follows:

$$
\begin{aligned}
D_{\text {total_ } 1} & =\left\{x:|x| \leq \sqrt{2 \Xi}, x=\varepsilon_{x}, \varepsilon_{y}, u_{e}, v_{e}, r_{e}\right\}, \\
D_{\text {total_2 }} & =\left\{\left\|\widetilde{w}_{i}\right\|:\left\|\widetilde{w}_{i}\right\| \leq \sqrt{2 \lambda_{\max }\left(\Gamma_{i}\right) \Xi}, i=u, v, r\right\}, \\
\Xi & =\min \left\{\left(\frac{C_{2}}{\rho_{1} \theta_{\rho_{1}}}\right)^{2 / p_{1}+1},\left(\frac{C_{2}}{8^{\left(1-q_{1}\right) / 2} \rho_{2} \theta_{\rho_{2}}}\right)^{2 / q_{1}+1}\right\}, \\
T_{\text {total }} & \leq \frac{2}{\rho_{1}\left(1-\theta_{\rho_{1}}\right)\left(1-p_{1}\right)}+\frac{2}{8^{\left(1-q_{1}\right) / 2} \rho_{2}\left(1-\theta_{\rho_{2}}\right)\left(q_{1}-1\right)},
\end{aligned}
$$

where $\theta_{\rho_{1}}, \theta_{\rho_{2}} \in(0,1)$ are positive constants.

In the light of Theorem 1 and the analyses of Steps 1 and 2 , the boundedness of $\varepsilon_{x}$ and $\varepsilon_{y}$ ensures that the position tracking errors $x_{e}$ and $y_{e}$ always satisfy their prescribed performance restraints. Furthermore, in terms of (21), we can conclude that the position tracking errors $x_{e}$ and $y_{e}$ will converge to a small region $D_{p}=D_{p 1} \cup D_{p 2}$ within a fixed time $T_{\text {total }}$, given by the following:

$$
\begin{aligned}
& D_{p 1}=\left\{x_{e}: \eta\left(T_{\text {total }}\right) S_{x}(-\sqrt{2 \Xi}) \leq x_{e} \leq \eta\left(T_{\text {total }}\right) S_{x}(\sqrt{2 \Xi})\right\}, \\
& D_{p 2}=\left\{y_{e}: \eta\left(T_{\text {total }}\right) S_{y}(-\sqrt{2 \Xi}) \leq y_{e} \leq \eta\left(T_{\text {total }}\right) S_{y}(\sqrt{2 \Xi})\right\},
\end{aligned}
$$

where $\eta\left(T_{\text {total }}\right)=\left(\eta_{0}-\eta_{\infty}\right) e^{-a T_{\text {total }}}+\eta_{\infty}$.

Remark 4. In the light of (58), we know that the coefficients $\rho_{1}, p_{1}, \rho_{2}$, and $q_{1}$ determine the precision of the tracking errors, namely, the small enough tracking errors are obtained by selecting the suitable coefficients. If $\varepsilon_{x}$ and $\varepsilon_{y}$ approach zero within a fixed time $T_{\text {total }}$ by choosing suitable coefficients, then the position tracking errors $x_{e}$ and $y_{e}$ approach zero within a fixed time based on (21) as well.
Remark 5. It is worth noting that the small tracking errors will bring about the large control energy so that the input saturation will occur in the control process. Accordingly, in practical applications, the coefficients $\rho_{1}, p_{1}, \rho_{2}$, and $q_{1}$ should be adjusted discreetly for obtaining superior control performance.

Remark 6. For dealing with uncertainty in the control system, many successful methods have been proposed by researchers. For example, the multiobjective optimal control method [37, 38], fuzzy approximation strategy [39], and neural approximation strategy [40] can effectively solve uncertainty in control problems and may yield low computational cost. However, an ARBFNN is used to approximate the model uncertainty in this paper to better combine with the fixed-time theory. In future work, we will combine the multiobjective optimal control method and fuzzy/neural approximation strategy to effectively deal with the uncertainty in the hovercraft's control system for obtaining the optimal control performance and lower computational cost in the real-time control process.

Remark 7. Through the efforts of researchers, the general prescribed performance constraint control method is improved positively such as $[41,42]$. The improved performance function was designed to overcome the existing prescribed performance constraint control method's shortcoming that the initial errors have to be known in advance for control designs. In this paper, it is the first time that fixed-time stability theory is introduced into the general prescribed performance control method. Therefore, the proposed method improved the convergence speed of the transformation errors, so that the convergence speed of the position tracking errors is also accelerated. Accordingly, this paper only uses a general prescribed performance control method that is better introduced into fixed-time stability theory, and the improved prescribed performance control method $[41,42]$ will be introduced into future work. 
TABLE 1: Main particulars of the hovercraft.

\begin{tabular}{lccc}
\hline$m_{0}(\mathrm{~kg})$ & 4000 & $J_{z 0}\left(\mathrm{~kg} \cdot \mathrm{m}^{2}\right)$ & $1.8 \times 10^{6}$ \\
\hline$J_{x 0}\left(\mathrm{~kg} \cdot \mathrm{m}^{2}\right)$ & $2.5 \times 10^{5}$ & $S_{\mathrm{PP}}\left(\mathrm{m}^{2}\right)$ & 45 \\
$S_{\mathrm{LP}}\left(\mathrm{m}^{2}\right)$ & 93 & $S_{\mathrm{HP}}\left(\mathrm{m}^{2}\right)$ & 260 \\
$g\left(\mathrm{~m} / \mathrm{s}^{2}\right)$ & 9.8 & $S_{c}\left(\mathrm{~m}^{2}\right)$ & 212 \\
$l_{s k}(\mathrm{~m})$ & 65 & $B_{c}(\mathrm{~m})$ & 8.9 \\
$\beta_{w}(\mathrm{deg})$ & 45 & $h_{m}(\mathrm{~m})$ & 2.4 \\
$l_{c}(\mathrm{~m})$ & 23.6 & $h(\mathrm{~m})$ & 1 \\
$H_{\text {hov }}(\mathrm{m})$ & 5.9 & $V_{w}(\mathrm{knot})$ & 5 \\
\hline
\end{tabular}

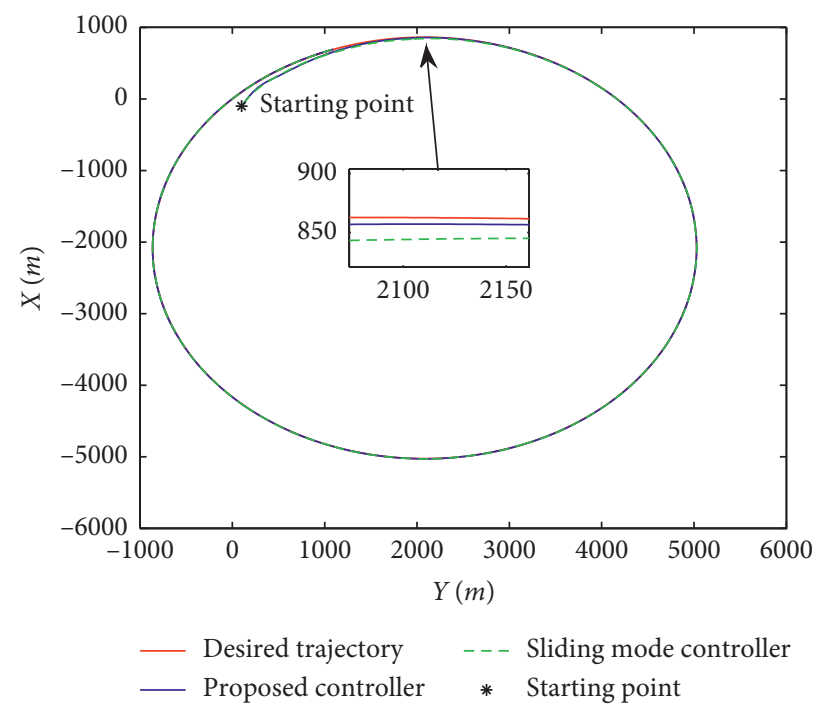

Figure 2: The actual and desired trajectory of the hovercraft.

\section{Simulation Results}

In this section, we complete the computer simulation experiments by comparison with the finite-time terminal sliding mode control method in [9] to verify the effectiveness and robustness of the proposed control method. In simulations, the hovercraft's main particulars are shown in Table $1[2,9]$.

The control parameters are set as $k_{p 11}=0.01$, $k_{p 12}=0.02, \quad k_{p 21}=0.001, \quad k_{p 22}=0.001, \quad k_{x}=0.0005$, $k_{y}=0.005, \quad p_{1}=0.7, \quad q_{1}=1.2, \quad k_{u}=1, \quad k_{v}=1.5, \quad k_{r}=2$, $k_{u 1}=0.1, \quad k_{u 2}=0.4, \quad k_{v 1}=1.2, \quad k_{v 2}=0.15, \quad k_{r 2}=3.5$, $k_{r 1}=0.02, \quad \alpha_{1}=\alpha_{2}=1, \beta_{1}=\beta_{2}=1, \eta_{0}=200, \quad \eta_{\infty}=2.2$, $a=0.02$, and $\bar{\delta}_{u}=\bar{\delta}_{v}=\bar{\delta}_{r}=0.1$. Since ARBFNN is utilized to approximate the unknown terms $f_{u}, f_{v}$, and $f_{r}$, the hidden node numbers for $\widehat{w}_{u}^{T} h_{u}\left(z_{u}\right), \widehat{w}_{v}^{T} h_{v}\left(z_{v}\right)$, and $\widehat{w}_{r}^{T} h_{r}\left(z_{r}\right)$ are set to $n=13$ and the centres $\phi_{l_{1}}\left(l_{1}=1, \ldots, n\right)$, $\phi_{l_{2}}\left(l_{2}=1, \ldots, n\right)$, and $\phi_{l_{3}}\left(l_{3}=1, \ldots, n\right)$ are evenly spaced in $[30,40],[-2,2]$, and $[-1,1]$, respectively. The widths are chosen as $\varepsilon_{l_{1}}=2, \varepsilon_{l_{2}}=1$, and $\varepsilon_{l_{2}}=1$, respectively.

The hovercraft's initial values are set as $x(0)=-100 \mathrm{~m}$, $y(0)=100 \mathrm{~m}, \quad \phi(0)=0, \quad \psi(0)=30^{\circ}, \quad u(0)=30$ knots, $v(0)=0, p(0)=0$, and $r(0)=0$.

The virtual surface vessel's parameters are set as $x_{d}(0)=0, \quad y_{d}(0)=0, \quad \psi_{d}(0)=45^{\circ}, \quad u_{d \text { set }}(t)=35$ knots, $v_{d \text { set }}(t)=0$, and $r_{d \text { set }}(t)=0.35^{\circ} / s$.
The total uncertainties suffered by the controlled system are characterized by the following formula:

$$
\left[\begin{array}{l}
f_{u} \\
f_{v} \\
f_{r}
\end{array}\right]=\left[\begin{array}{ll}
\frac{2 u \sin (0.05 t)}{\left(10 m_{0}\right)} & \\
\frac{v \cos (0.03 t)}{m_{0}} & \\
& \frac{10^{2} r \cos (0.02 t)}{J_{z 0}}
\end{array}\right]\left[\begin{array}{l}
b_{1} \\
b_{2} \\
b_{3}
\end{array}\right],
$$

where $b=\left[\begin{array}{lll}b_{1} & b_{2} & b_{3}\end{array}\right]^{T} \in R^{3}$ and $\dot{b}=-T^{-1} b+A w_{n}$ signifies the first-order Markov process with $w_{n} \in R^{3}$ representing the zero-mean Gaussian white noises' vector, and the rest coefficients of the first-order Markov process can be expressed as follows:

$$
\begin{aligned}
b(0) & =\left[2 \times 10^{4}, 2 \times 10^{4}, 2 \times 10^{4}\right]^{T}, \\
T & =\operatorname{diag}\left(10^{3}, 10^{3}, 10^{3}\right), \\
A & =\operatorname{diag}\left(1 \times 10^{4}, 1 \times 10^{4}, 1 \times 10^{4}\right) .
\end{aligned}
$$

The simulation results of the two different methods with and without prescribed performance constraints are shown 


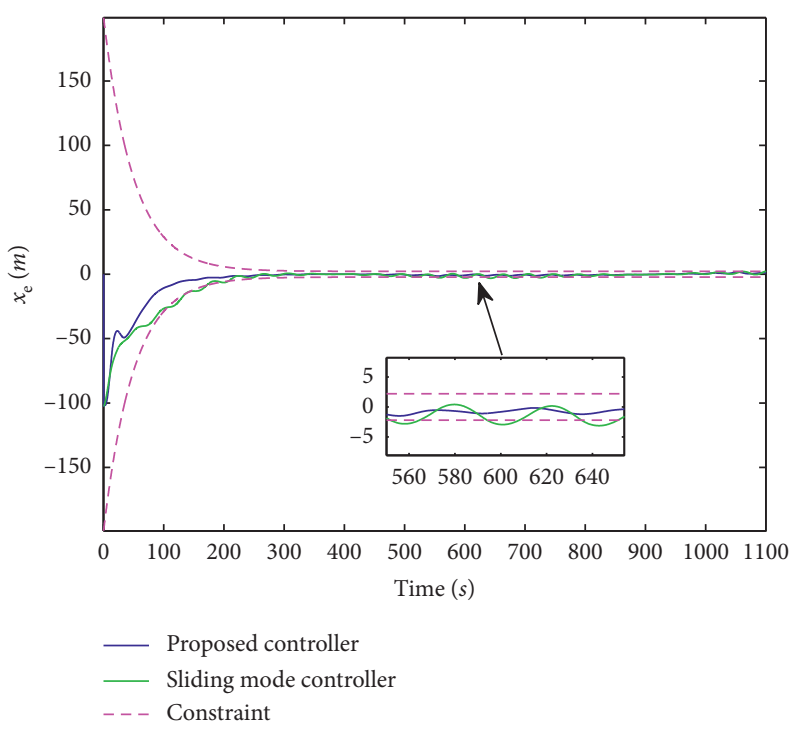

(a)

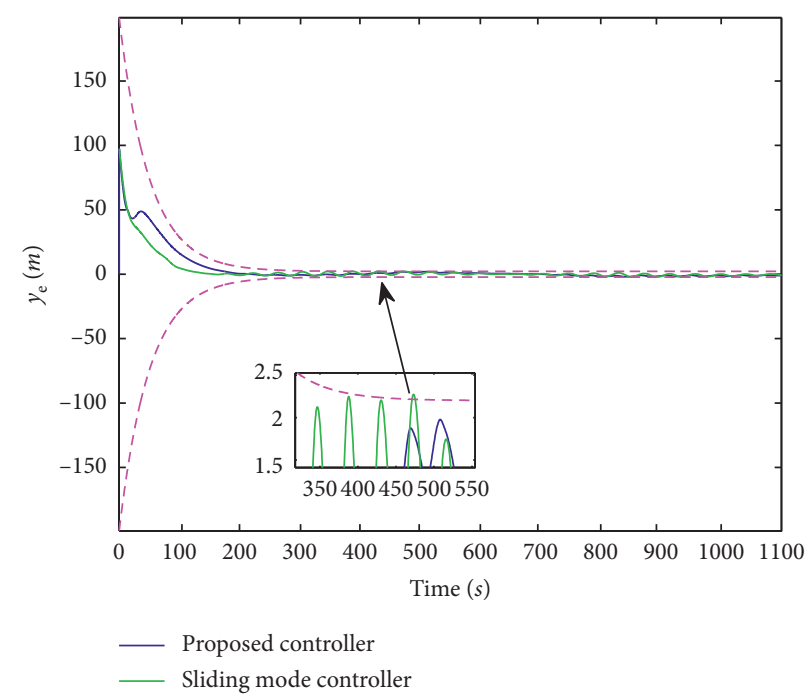

(b)

Figure 3: Position tracking errors.


(a)

(b)

Figure 4: Input of two methods.


(a)

(b)

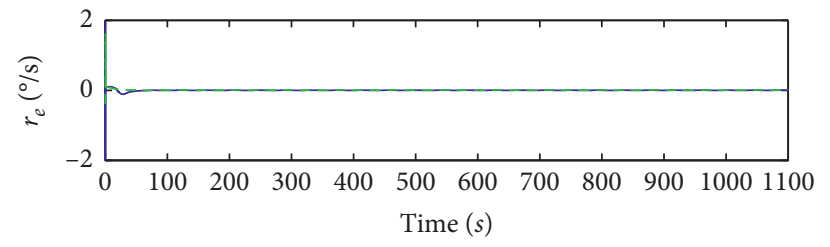

_ Proposed controller

_. - Sliding mode controller

(c)

FIgURE 5: Velocity and yaw angular velocity tracking errors. 


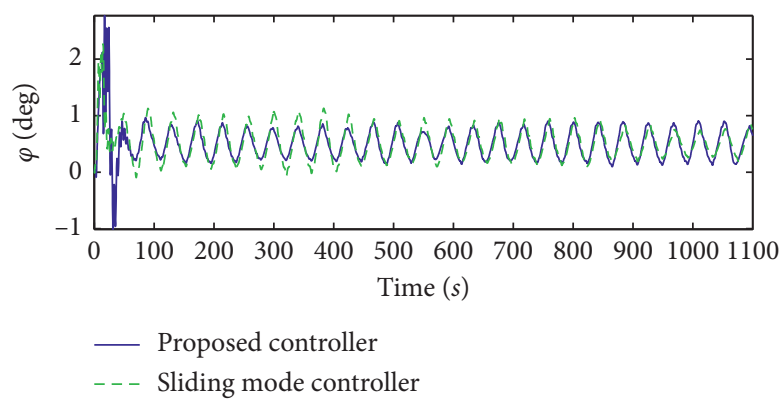

(a)

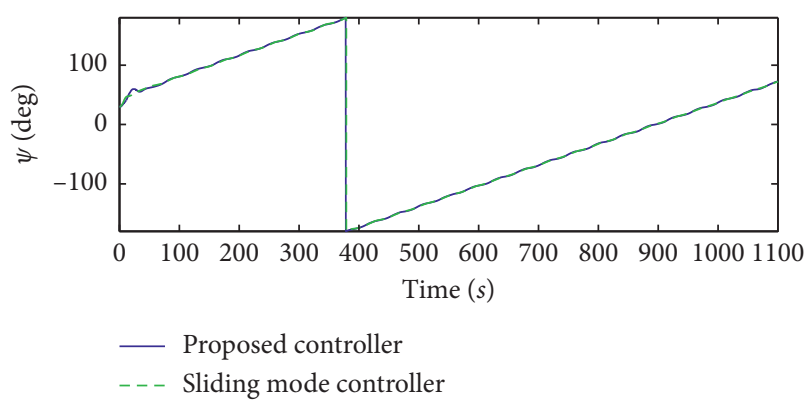

(b)

FIGURE 6: Roll angular and yaw angular states.



(a)

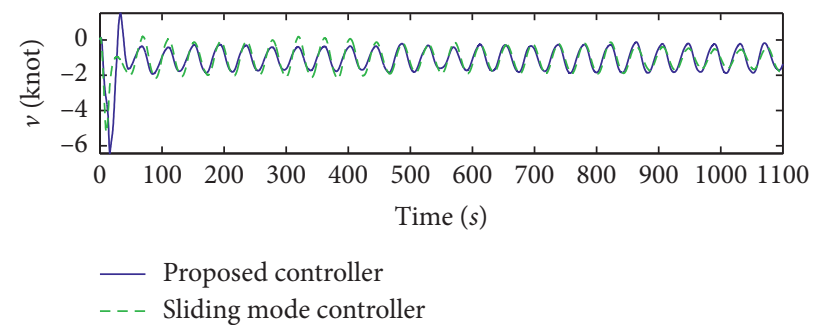

(b)

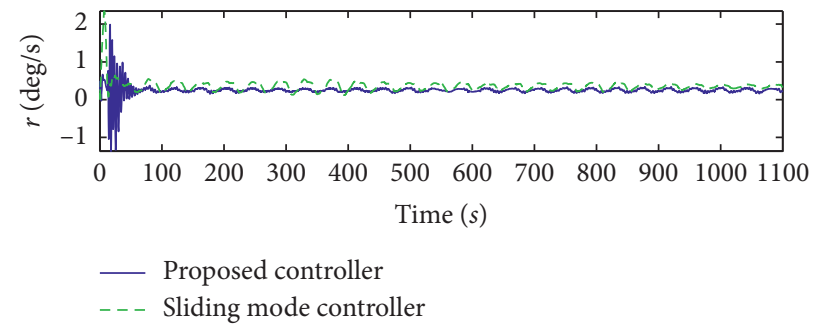

(c)

Figure 7: Velocity and yaw angular velocity states.

in Figures 2-7 and labelled as proposed controller and sliding mode controller, respectively. Figure 2 shows the hovercraft can follow the virtual surface vessel despite the presence of the system uncertainties. The desired trajectory is constructed by a circle, which can represent practical significance in the problem of path following or trajectory tracking. Furthermore, from Figure 3, it is obvious that the proposed method can ensure the prespecified transient and steady performance of the position tracking errors. However, the constraints on the position tracking errors are violated under the sliding mode controller.

Subsequently, from Figures 3 and 5, it is observed that the controllers designed in this paper are effective and can ensure that the position tracking error, velocity tracking errors, and the yaw angular velocity tracking error converge to a very small region around zero. Further observation shows that the proposed controller has a faster convergence speed and higher tracking precision than the sliding mode controller. The curves of the roll angle, yaw angle, and velocity states evolved with respect to time are shown in
Figures 6 and 7. These two controllers' control inputs are shown in Figure 4. All simulation results illustrate the superiority and robustness of the proposed method.

\section{Conclusion}

Driven by the practical requirements for the underactuated hovercraft to sail in confined channels, the Lyapunov direct method and fixed-time stability theory-based trajectory tracking control algorithm with a prescribed performance is proposed for the hovercraft's motion control while being subjected to model uncertainties and external disturbances. The prescribed performance bound technique and fixedtime stability theory, which are integrated into Lyapunov stability synthesis, successfully guarantee that the position tracking errors of the hovercraft remain within the prespecified performance limits. It is shown that with the proposed control strategy, the requirement of the prespecified performance is always not violated and all tracking errors are not only uniformly ultimately bounded but also 
fixed-time convergent to an arbitrarily small region containing zero. In the future, we will consider that the velocity states are constrained to guarantee the safe sailing of the high-speed hovercraft.

\section{Data Availability}

The data in this paper are derived from the International Cooperation Projects. Hence, the data are private.

\section{Conflicts of Interest}

The authors declare that they have no conflicts of interest.

\section{Acknowledgments}

This work was supported by the National Nature Science Foundation of China (grant nos. 51309062 and 52071112).

\section{References}

[1] L. Yun and A. Bliault, Theory \& Design of Air Cushion Craft, Theory Des. Air Cushion Cr, pp. 577-611, Elsevier, Amsterdam, Netherlands, 2000, http://www.sciencedirect.com/ science/article/pii/B9780340676509500176.

[2] M. Fu, S. Gao, C. Wang, and M. Li, "Design of driver assistance system for air cushion vehicle with uncertainty based on model knowledge neural network," Ocean Engineering, vol. 172, pp. 296-307, 2019.

[3] H. Fu, Analysis and Consideration on Safety of All-Lift Hovercraft, pp. 1-3, Ship and Boat, Shanghai, China, 2008.

[4] M. Cohen, T. Miloh, and G. Zilman, "Wave resistance of a hovercraft moving in water with nonrigid bottom," Ocean Engineering, vol. 28, no. 11, pp. 1461-1478, 2001.

[5] V. M. Kozin and A. V. Pogorelova, "Variation in the wave resistance of an amphibian air-cushion vehicle moving over a broken-ice field," Journal of Applied Mechanics and Technical Physics, vol. 48, no. 1, pp. 80-84, 2007.

[6] X. Lin, J. Nie, Y. Jiao, K. Liang, and H. Li, "Adaptive fuzzy output feedback stabilization control for the underactuated surface vessel," Applied Ocean Research, vol. 74, pp. 40-48, 2018.

[7] C.-Z. Pan, X.-Z. Lai, S. X. Yang, and M. Wu, "A biologically inspired approach to tracking control of underactuated surface vessels subject to unknown dynamics," Expert Systems with Applications, vol. 42, no. 4, pp. 2153-2161, 2015.

[8] J. Xu, M. Wang, and L. Qiao, "Dynamical sliding mode control for the trajectory tracking of underactuated unmanned underwater vehicles," Ocean Engineering, vol. 105, pp. 54-63, 2015.

[9] M. Fu, S. Gao, C. Wang, and M. Li, "Human-centered automatic tracking system for underactuated hovercraft based on adaptive chattering-free full-order terminal sliding mode control," IEEE Access, vol. 6, pp. 37883-37892, 2018.

[10] W.-B. Xie, Y.-L. Wang, J. Zhang, and M.-Y. Fu, "Novel separation principle based $\$ \mathrm{H}_{-}$linfty\$ observer-controller design for a class of T-S fuzzy systems," IEEE Transactions on Fuzzy Systems, vol. 26, no. 6, pp. 3206-3221, 2018.

[11] C. P. Bechlioulis and G. A. Rovithakis, "Prescribed performance adaptive control for multi-input multi-output affine in the control nonlinear systems," IEEE Transactions on Automatic Control, vol. 55, no. 5, pp. 1220-1226, 2010.
[12] W. Wang and C. Wen, "Adaptive actuator failure compensation control of uncertain nonlinear systems with guaranteed transient performance," Automatica, vol. 46, no. 12, pp. 2082-2091, 2010.

[13] C. Wang and Y. Lin, "Adaptive dynamic surface control for MIMO nonlinear time-varying systems with prescribed tracking performance," International Journal of Control, vol. 88, no. 4, pp. 832-843, 2015.

[14] J. Na, Q. Chen, X. Ren, and Y. Guo, “Adaptive prescribed performance motion control of servo mechanisms with friction compensation," IEEE Transactions on Industrial Electronics, vol. 61, no. 1, pp. 486-494, 2014.

[15] W. Meng, Q. Yang, and Y. Sun, "Guaranteed performance control of DFIG variable-speed wind turbines," IEEE Transactions on Control Systems Technology, vol. 24, no. 6, pp. 2215-2223, 2016.

[16] X. Bu, X. Wu, J. Huang, and D. Wei, "Robust estimation-free prescribed performance back-stepping control of airbreathing hypersonic vehicles without affine models," International Journal of Control, vol. 89, no. 11, pp. 2185-2200, 2016.

[17] E. Psomopoulou, A. Theodorakopoulos, Z. Doulgeri, and G. A. Rovithakis, "Prescribed performance tracking of a variable stiffness actuated robot," IEEE Transactions on Control Systems Technology, vol. 23, no. 5, pp. 1914-1926, 2015.

[18] W. Wang, J. Huang, and C. Wen, "Prescribed performance bound-based adaptive path-following control of uncertain nonholonomic mobile robots," International Journal of Adaptive Control and Signal Processing, vol. 31, no. 5, pp. 805-822, 2017.

[19] X. Bu, "Envelope-constraint-based tracking control of airbreathing hypersonic vehicles," Aerospace Science and Technology, vol. 95, p. 105429, 2019.

[20] X. Bu, Y. Xiao, and H. Lei, "An adaptive critic design-based fuzzy neural controller for hypersonic vehicles: predefined behavioral nonaffine control," IEEE/ASME Transactions on Mechatronics, vol. 24, no. 4, pp. 1871-1881, 2019.

[21] S. Zhao, X. Li, X. Bu, and D. Zhang, "Prescribed performance tracking control for hypersonic flight vehicles with model uncertainties," International Journal of Aerospace Engineering, vol. 2019, Article ID 3505614, 11 pages, 2019.

[22] X. Bu, G. He, and D. Wei, "A new prescribed performance control approach for uncertain nonlinear dynamic systems via back-stepping," Journal of the Franklin Institute, vol. 355, no. 17, pp. 8510-8536, 2018.

[23] X. Bu, "Guaranteeing prescribed performance for airbreathing hypersonic vehicles via an adaptive non-affine tracking controller," Acta Astronautica, vol. 151, pp. 368-379, 2018.

[24] T. Gao, J. Huang, Y. Zhou, and Y.-D. Song, "Robust adaptive tracking control of an underactuated ship with guaranteed transient performance," International Journal of Systems Science, vol. 48, no. 2, pp. 272-279, 2017.

[25] C. P. Bechlioulis, G. C. Karras, S. Heshmati-Alamdari, and K. J. Kyriakopoulos, "Trajectory tracking with prescribed performance for underactuated underwater vehicles under model uncertainties and external disturbances," IEEE Transactions on Control Systems Technology, vol. 25, no. 2, pp. 429-440, 2017.

[26] C. Chen, S. Zhu, Y. Wei, and C. Chen, "Finite-time stability of delayed memristor-based fractional-order neural networks," IEEE Transactions on Cybernetics, vol. 50, no. 4, pp. 16071616, 2020. 
[27] X. Jin, "Adaptive fixed-time control for MIMO nonlinear systems with asymmetric output constraints using universal barrier functions," IEEE Transactions on Automatic Control, vol. 64, no. 7, pp. 3046-3053, 2019.

[28] B. Jiang, Q. Hu, and M. I. Friswell, "Fixed-time attitude control for rigid spacecraft with actuator saturation and faults," IEEE Transactions on Control Systems Technology, vol. 24, no. 5, pp. 1892-1898, 2016.

[29] L. Zhang, C. Wei, L. Jing, and N. Cui, "Fixed-time sliding mode attitude tracking control for a submarine-launched missile with multiple disturbances," Nonlinear Dynamics, vol. 93, no. 4, pp. 2543-2563, 2018.

[30] C. Dong, Y. Liu, and Q. Wang, "Barrier Lyapunov function based adaptive finite-time control for hypersonic flight vehicles with state constraints," ISA Transactions, vol. 96, pp. 163-176, 2020.

[31] Z. Zuo and L. Tie, "Distributed robust finite-time nonlinear consensus protocols for multi-agent systems," International Journal of Systems Science, vol. 47, no. 6, pp. 1366-1375, 2016.

[32] M. Fu, T. Wang, and C. Wang, "Adaptive neural-based finitetime trajectory tracking control for underactuated marine surface vessels with position error constraint," IEEE Access, vol. 7, pp. 16309-16322, 2019.

[33] A. Polyakov, "Nonlinear feedback design for fixed-time stabilization of linear control systems," IEEE Transactions on Automatic Control, vol. 57, no. 8, pp. 2106-2110, 2012.

[34] Z. Zheng, M. Feroskhan, and L. Sun, "Adaptive fixed-time trajectory tracking control of a stratospheric airship," ISA Transactions, vol. 76, pp. 134-144, 2018.

[35] X. Jin, "Fault tolerant finite-time leader-follower formation control for autonomous surface vessels with LOS range and angle constraints," Automatica, vol. 68, pp. 228-236, 2016.

[36] Z. Zheng and M. Feroskhan, "Path following of a surface vessel with prescribed performance in the presence of input saturation and external disturbances," IEEE/ASME Transactions on Mechatronics, vol. 22, no. 6, pp. 2564-2575, 2017.

[37] F. Logist, B. Houska, M. Diehl, and J. F. Van Impe, "Robust multi-objective optimal control of uncertain (bio) chemical processes," Chemical Engineering Science, vol. 66, no. 20, pp. 4670-4682, 2011.

[38] C. Liu, Z. Gong, K. L. Teo, J. Sun, and L. Caccetta, "Robust multi-objective optimal switching control arising in 1, 3propanediol microbial fed-batch process," Nonlinear Analysis: Hybrid Systems, vol. 25, pp. 1-20, 2017.

[39] X. Bu and Q. Qi, "Fuzzy optimal tracking control of hypersonic flight vehicles via single-network adaptive critic design," IEEE Transactions on Fuzzy Systems, vol. 2020, Article ID 3036706, 1 page, 2020.

[40] X. Bu, "Air-breathing hypersonic vehicles funnel control using neural approximation of non-affine dynamics," IEEE/ ASME Transactions on Mechatronics, vol. 23, no. 5, pp. 2099-2108, 2018.

[41] X. Bu, Y. Xiao, and K. Wang, "A prescribed performance control approach guaranteeing small overshoot for airbreathing hypersonic vehicles via neural approximation," Aerospace Science and Technology, vol. 71, pp. 485-498, 2017.

[42] X. Bu, X. Wu, F. Zhu, J. Huang, Z. Ma, and R. Zhang, "Novel prescribed performance neural control of a flexible airbreathing hypersonic vehicle with unknown initial errors," ISA Transactions, vol. 59, pp. 149-159, 2015. 